\title{
BOUNDARY RIGIDITY WITH PARTIAL DATA
}

\author{
PLAMEN STEFANOV, GUNTHER UHLMANN, AND ANDRAS VASY
}

\section{INTRODUCTION AND MAIN RESULTS}

Travel time tomography deals with the problem of determining the sound speed or index of refraction of a medium by measuring the travel times of waves going through the medium. This type of inverse problem, also called the inverse kinematic problem, arose in geophysics in an attempt to determine the substructure of the Earth by measuring the travel times of seismic waves at the surface. We consider an anisotropic index of refraction, i.e., a sound speed depending on the direction. The Earth is generally isotropic. However, more recently it has been realized, by measuring these travel times, that the inner core of the Earth exhibits anisotropic behavior with the fast direction parallel to the Earth's spin axis; see [5]. In the human body, muscle tissue is anisotropic. As a model of anisotropy, we consider a Riemannian metric $g=\left(g_{i j}\right)$. The problem is to determine the metric from the lengths of geodesics joining points on the boundary.

This leads to the general question of whether given a compact Riemannian manifold with boundary $(M, g)$ one can determine the Riemannian metric in the interior knowing the boundary distance function joining points on the boundary $d_{g}(x, y)$, with $x, y \in \partial M$. This is known as the boundary rigidity problem. Of course, isometries preserve distance, so that the boundary rigidity problem is whether two metrics that have the same boundary distance function are the same up to isometry fixing the boundary. Examples can be given of manifolds that are not boundary rigid. Such examples show that the boundary rigidity problem should be considered under some restrictions on the geometry of the manifold. The most usual of such restrictions is simplicity of the metric. A Riemannian manifold $(M, g)$ (or the metric $g$ ) is called simple if the boundary $\partial M$ is strictly convex (positive second fundamental form) and any two points $x, y \in M$ are joined by a unique minimizing geodesic. Michel conjectured [21] that every simple compact Riemannian manifold with boundary is boundary rigid.

Simple surfaces with boundary are boundary rigid [29. In higher dimensions, simple Riemannian manifolds with boundary are boundary rigid under some a priori constant curvature on the manifold or special symmetries [2, 13]. Several local results near the Euclidean metric are known [3, 19, 39. The most general result in this direction is the generic local (with respect to the metric) one proven in [41]. Surveys of some of the results can be found in [8, 16, 42].

Received by the editors November 13, 2013.

2010 Mathematics Subject Classification. Primary 53C24, 35R30.

The first author was partly supported by NSF Grant DMS-1301646.

The second author was partly supported by NSF Grants CMG-1025259 and DMS-1265958, The Fondation Mathématiques de Paris, and a Simons fellowship.

The third author was partly supported by NSF Grants CMG-1025259 and DMS-1068742. 
In this paper, we consider the boundary rigidity problem in the class of metrics conformal to a given one and with partial (local) data; that is, we know the boundary distance function for points on the boundary near a given point. Partial data problems arise naturally in applications since in many cases one does not have access to the whole boundary. We prove the first result on the determination of the conformal factor locally near the boundary from partial data without assuming analyticity. We develop a novel method to attack partial data nonlinear problems that will likely have other applications.

We now describe the known results with full data on the boundary. Let us fix the metric $g_{0}$ and let $c$ be a positive smooth function on the compact manifold with boundary $M$. The problem is whether we can determine $c$ from $d_{c^{-2} g_{0}}(x, y), x, y \in$ $\partial M$. Notice that in this case the problem is not invariant under changes of variables that are the identity at the boundary so that we expect to be able to recover $c$ under appropriate a priori conditions. This was proven by Mukhometov in two dimensions [23], and in 24] in higher dimensions for the case of simple metrics. Of particular importance in applications is the case of an isotropic sound speed that is when we are in a bounded domain of Euclidean space and $g_{0}$ is the Euclidean metric. This is the isotropic case. This problem was considered by Herglotz [14] and Wieckert and Zoeppritz [51] for the case of a spherical symmetric sound speed. They found a formula to recover the sound speed from the boundary distance function assuming $\frac{\mathrm{d}}{\mathrm{d} r}\left(\frac{r}{c(r)}\right)>0$. Notice that this condition is equivalent to the existence of a strictly convex foliation and is more general than simplicity; see Section 6 .

From now on we will call $d$ the function $d_{c^{-2} g_{0}}$. Below, $\tilde{d}$ is related to $d_{\tilde{c}^{-2} g_{0}}$.

The partial data problem, which we will also call the local boundary rigidity problem 11 in this case is whether knowledge of the distance function on part of the boundary determines the sound speed $c$ locally. Given another smooth $\tilde{c}$, here and below we define $\tilde{L}, \tilde{\ell}$, and $\tilde{d}$ in the same way but related to $\tilde{c}$. We prove the following uniqueness result.

Theorem 1.1. Let $n=\operatorname{dim} M \geq 3$, let $c>0, \tilde{c}>0$ be smooth, and let $\partial M$ be strictly convex with respect to both $g=c^{-2} g_{0}$ and $\tilde{g}=\tilde{c}^{-2} g_{0}$ near a fixed $p \in \partial M$. Let $d\left(p_{1}, p_{2}\right)=\tilde{d}\left(p_{1}, p_{2}\right)$ for $p_{1}, p_{2}$ on $\partial M$ near $p$. Then $c=\tilde{c}$ in $M$ near $p$.

As mentioned earlier, this is the only known result for the boundary rigidity problem with partial data except in the case that the metrics are assumed to be real-analytic [19]. The latter follows from determination of the jet of the metric at a convex point from the distance function known near $p$.

The boundary rigidity problem is closely connected to the lens rigidity one. To define the latter, we first introduce the manifolds $\partial_{ \pm} S M$, defined as the sets of all vectors $(x, v)$ with $x \in \partial M, v$ unit in the metric $g$, and pointing outside/inside $M$. We define the scattering relation

$$
L: \partial_{-} S M \longrightarrow \partial_{+} S M
$$

in the following way: for each $(x, v) \in \partial_{-} S M, L(x, v)=(y, w)$, where $(y, w)$ are the exit point and direction, if they exist, of the maximal unit speed geodesic $\gamma_{x, v}$ in the metric $g$, issued from $(x, v)$. Let

$$
\ell: \partial_{-} S M \longrightarrow \mathbb{R} \cup \infty
$$

\footnotetext{
${ }^{1}$ It is local in the sense that $d(x, y)$ is known locally and depends on $c$ locally; the term local has been used before to indicate that the metric is a priori close to a fixed one.
} 
be its length, possibly infinite. If $\ell<\infty$, we call $M$ non-trapping. The maps $(L, \ell)$ together are called lens relation (or lens data).

The lens rigidity problem is whether the scattering relation $L$ (and possibly, $\ell$ ) determine $g$ (and the topology of $M$ ) up to an isometry as above. The lens rigidity problem with partial data for a sound speed is whether we can determine the speed near some $p$ from $L$ known near the unit sphere $S_{p} \partial M$ considered as a subset of $\partial_{-} S M$, i.e., for vectors with base points close to $p$ and directions pointing into $M$ close to ones tangent to $\partial M$. For general metrics, we want to recover isometric copies of the metrics locally, as above.

We assume that $\partial M$ is strictly convex at $p \in \partial M$ with respect to (w.r.t.) $g$. Then the boundary rigidity and the lens rigidity problems with partial data are equivalent: knowing $d$ near $(p, p)$ is equivalent to knowing $L$ in some neighborhood of $S_{p} \partial M$. The size of that neighborhood, however, depends on a priori bounds of the derivatives of the metrics with which we work. This equivalence was first noted by Michel [21], since the tangential gradients of $d(x, y)$ on $\partial M \times \partial M$ give us the tangential projections of $-v$ and $w$; see also [38, Section 2]. Note that local knowledge of $\ell$ is not needed for the lens rigidity problem 2 and in fact, $\ell$ can be recovered locally from either $d$ or $L$; see, for example, the proof of Theorem 5.2 .

Vargo [50] proved that real-analytic manifolds satisfying an additional mild condition are lens rigid. Croke has shown that if a manifold is lens rigid, a finite quotient of it is also lens rigid [8]. He has also shown that the torus is lens rigid [4]. Uhlmann and Stefanov have shown lens rigidity locally near a generic class of non-simple manifolds [44. In a recent work, Guillarmou [12] proved that in two dimensions, one can determine from the lens relation the conformal class of the metric if the trapped set is hyperbolic and there are no conjugate points. He also proved deformation lens rigidity in higher dimensions under the same assumptions. The only result we know for the lens rigidity problem with incomplete (but not local) data is for real-analytic metric and metric close to them satisfying the micolocal condition in the next sentence [44]. While in [44, the lens relation is assumed to be known on a subset only, the geodesics issued from that subset cover the whole manifold, and their conormal bundle is required to cover $N^{*} M$. In contrast, in this paper, we have localized information.

We state below an immediate corollary of our main result for this problem. For the partial data problem instead of assuming $d=\tilde{d}$ locally, we can assume that $L=\tilde{L}$ in a neighborhood of $S_{p} \partial M$. To reduce this problem to Theorem 1.1 directly, we need to assume first that $c=\tilde{c}$ on $\partial M$ near $p$ to make the definition of $\partial_{ \pm} S M$ independent of the choice of the speed but, in fact, one can redefine the lens relation in a way to remove that assumption; see [44.

Theorem 1.2. Let $M, c, \tilde{c}$ be as in Theorem 1.1 with $c=\tilde{c}$ on $\partial M$ near $p$. Let $L=\tilde{L}$ near $S_{p} \partial M$. Then $c=\tilde{c}$ in $M$ near $p$.

Remark 1.3. The theorem or its corollary does not preclude the existence of an infinite set of speeds $c_{j}$ all having the same boundary distance function (or lens data) in $U \times U$, where $U \subset \partial M$ is some fixed small set but not coinciding in any fixed neighborhood of $p$. In principle, this may happen when the maximal neighborhood of $U$, which can be covered with strictly convex surfaces, which continuously deform $U$, shrinks when $j \rightarrow \infty$. Then the theorem does not imply existence of a fixed

\footnotetext{
${ }^{2}$ If only $L$ is given, then the problem is called scattering rigidity in some works
} 
neighborhood of $p$, where all speeds are equal. If one assumes that a priori, the sound speeds have uniformly bounded derivatives of some finite order near $p$, this situation does not arise, and this case is covered by Theorem 5.2 below.

The linearization of the boundary rigidity and lens rigidity problem is the tensor tomography problem, i.e., recovery of a tensor field up to "potential fields" from integrals along geodesics joining points on the boundary. It has been extensively studied in the literature for both simple and non-simple manifolds $[1,11,22,26,28$, 30, 32, 34, 36, 40, 43, 46]. See the book [33] and [28, for a recent survey. The local tensor tomography problem has been considered in [17. for functions and realanalytic metrics and in 18 for tensors of order two and real-analytic metrics. Those results can also be thought of as support theorems of Helgason type. The only known results for the local problem for smooth metrics and integrals of functions is [49].

Now we use a layer stripping type argument to obtain a global result which is different from Mukhometov's for simple manifolds.

Definition 1.1. Let $(M, g)$ be a compact Riemannian manifold with boundary. We say that $M$ satisfies the foliation condition by strictly convex hypersurfaces if $M$ is equipped with a smooth function $\rho: M \rightarrow[0, \infty)$ with level sets $\Sigma_{t}=\rho^{-1}(t), t<T$ with some $T>0$ are strictly convex viewed from $\rho^{-1}((0, t))$ for $g, d \rho$ is non-zero on these level sets, and $\Sigma_{0}=\partial M$ and $M \backslash \cup_{t \in[0, T)} \Sigma_{t}$ has an empty interior.

The statement of the global result on lens rigidity is as follows.

Theorem 1.4. Let $n=\operatorname{dim} M \geq 3$, let $c>0, \tilde{c}>0$ be smooth and equal on $\partial M$, let $\partial M$ be strictly convex with respect to both $g=c^{-2} g_{0}$ and $\tilde{g}=\tilde{c}^{-2} g_{0}$. Assume that $M$ can be foliated by strictly convex hypersurfaces for $g$. Then if $L=\tilde{L}$ on $\partial_{-} S M$, we have $c=\tilde{c}$ in $M$.

A more general foliation condition under which the theorem would still hold is formulated in [45]; see also Definition 5.1 below. In particular, $\Sigma_{0}$ does not need to be $\partial M$, and one can have several such foliations with the property that the closure of their union is $M$. If we can foliate only some connected neighborhood of $\partial M$, we would get $c=\tilde{c}$ there. Next, it is enough to require that $M \backslash \cup_{t \in[0, T)} \Sigma_{t}$ is simple (or that it is included in a simple submanifold; see the proof of Theorem 1.4 and Figure 2in Section 4) to prove $c=\tilde{c}$ in $\cup_{t \in[0, T)} \Sigma_{t}$ first, and then use Mukhometov's results to complete the proof. The class of manifolds we get in this way is larger than the simple ones and can have conjugate points.

Speeds not necessarily radial (with $g_{0}$ the Euclidean metric) under the condition considered by Herglotz and Wieckert and Zoeppritz satisfy the foliation condition of the theorem; see also Section 6. Other examples of non-simple metrics that satisfy the condition are the tubular neighborhood of a closed geodesic in negative curvature. These have trapped geodesics. It follows from the result of [31], that manifolds with no focal points satisfy the foliation condition. It would be interesting to know whether this is also the case for simple manifolds. As it was mentioned earlier, manifolds satisfying the foliation condition are not necessarily simple.

The linearization of the non-linear problem with partial data considered in Theorem 1.1 was considered in 49, where uniqueness and stability were shown. This corresponds to integrating functions along geodesics joining points in a neighborhood of $p$. The method of proof of Theorem 1.1 relies on using an identity proven 
in 39 to reduce the problem to a "pseudo-linear" one: to show uniqueness when one integrates the function $f=c^{2}-\tilde{c}^{2}$ and its derivatives on the geodesics for the metric $g$ joining points near $p$, with weight depending non-linearly on both $g$ and $\tilde{g}$. Notice that this is not a proof by linearization, and unlike the problem with full data, an attempt to do such a proof by linearization is connected with essential difficulties. The proof of uniqueness for this linear transform follows the method of [49] introducing an artificial boundary and using Melrose's scattering calculus. In Section 2, we do the reduction to a "pseudo-linear problem," and in Section 3, we show uniqueness for the pseudo-linear problem. In Section 4, we finish the proofs of the main theorems.

We also prove Hölder conditional stability estimates related to the uniqueness theorems above. In the case of data on the whole boundary, such an estimate was proved in [41, Section 7] for simple manifolds and metrics not necessarily conformal to each other. Below, the $C^{k}$ norm is defined in a fixed coordinate system. The next theorem is a local stability result, corresponding to the local uniqueness result in Theorem 1.1 .

Theorem 1.5. There exists $k>0$ and $0<\mu<1$ with the following property. For any $0<c_{0} \in C^{k}(M), p \in \partial M$, and $A>0$, there exists $\varepsilon_{0}>0$ and $C>0$ with the property that for any two positive $c$, $\tilde{c}$ with

$$
\left\|c-c_{0}\right\|_{C^{2}}+\left\|\tilde{c}-c_{0}\right\|_{C^{2}} \leq \varepsilon_{0}, \quad \text { and } \quad\|c\|_{C^{k}}+\|\tilde{c}\|_{C^{k}} \leq A,
$$

and for any neighborhood $\Gamma$ of $p$ on $\partial M$, we have the stability estimate

$$
\|c-\tilde{c}\|_{C^{2}(U)} \leq C\|d-\tilde{d}\|_{C(\Gamma \times \Gamma)}^{\mu}
$$

for some neighborhood $U$ of $p$ in $M$.

In Theorem [5.2, we prove Hölder conditional stability estimates of global type as well, which can be considered as a "stable version" of Theorem 1.4 .

The plan of the paper is as follows. The reduction to a pseudo-linear problem is done in Section 2. In Section 3, we present linear analysis using the scattering calculus. The main result there is Proposition 3.3, which is of its own interest as well. The proofs of the three uniqueness theorems are in Section 4 . In Section 5, we prove the local stability result in Theorem 1.5 and the global Theorem 5.2. As an application of our results, we revisit the class of speeds studied by Herglotz [14] and Wieckert and Zoeppritz 51 in Section 6 without assuming that they are radial, and we prove that they are lens rigid. In particular, we show that their condition (6.1) is equivalent to the requirement that the Euclidean spheres are strictly convex for the metric $c^{-2} \mathrm{~d} x^{2}$; therefore, it is a foliation condition.

\section{ReduCing the NON-LINEAR PROBlem to A PSEUdO-LINEAR ONE}

We recall the known fact [19] that one can determine the jet of $c$ at any boundary point $p$ at which $\partial M$ is convex (not necessarily strictly) from the distance function $d$ known near $(p, p)$. For a more general result not requiring convexity, see 44]. Since the result in [19] is formulated for general metrics, and the reconstruction of the jet is in boundary normal coordinates, we repeat the proof in this (simpler) situation of recovery of a conformal factor. As in [19], we say that $\partial M$ is convex near $p \in \partial M$, if for any two distinct points $p_{1}, p_{2} \in \partial M$, close enough to $p$, there exists a geodesic $\gamma:[0,1] \rightarrow M$ joining them such that its length is $d\left(p_{1}, p_{2}\right)$ and all 
the interior of $\gamma$ belongs to the interior of $M$. Of course, strict convexity (positive second fundamental form at $p$ ) implies convexity.

Lemma 2.1. Let $c$ and $\tilde{c}$ be smooth and let $\partial M$ be convex at $p$ with respect to $g$ and $\tilde{g}$. Let $d=\tilde{d}$ near $(p, p)$. Then $\partial^{\alpha} c=\partial^{\alpha} \tilde{c}$ on $\partial M$ near $p$ for any multi-index $\alpha$.

Proof. Let $V \subset \partial M$ be a neighborhood of $p$ on $\partial M$ such that for any $p_{1}, p_{2} \in V$, we have the property guaranteeing convexity at $p$. Let $x^{n}$ be a boundary normal coordinate related to $g$; i.e., $x^{n}(q)=\operatorname{dist}(q, \partial M)$, and $x^{n} \geq 0$ in $M$. We can complete $x^{n}$ to a local coordinate system $\left(x^{\prime}, x^{n}\right)$, where $x^{\prime}$ parameterizes $\partial M$ near $p$.

It is enough to prove

$$
\partial_{x^{n}}^{k} c=\partial_{x^{n}}^{k} \tilde{c} \quad \text { in } V, k=0,1, \ldots
$$

For $k=0$, this follows easily by taking the limit in $d(p, q)=\tilde{d}(p, q), \partial M \ni q \rightarrow p$; and this can be done for any $p \in V$. Let $l$ be the first value of $k$ for which (2.1) fails. Without loss of generality, we may assume that it fails at $p$, and $\partial_{x^{n}}^{l}(c-\tilde{c})>0$ at $p$. Then $\partial_{x^{n}}^{k}(c-\tilde{c})=0$ in $V, k=0, \ldots, l-1$. Consider the Taylor expansion of $c-\tilde{c}$ w.r.t. $x^{n}$ with $x^{\prime}$ close enough to $x^{\prime}(p)$. We get $c-\tilde{c}>0$ in some neighborhood of $p$ in $M$ minus the boundary.

Now, let $\gamma(p, q)$ be a minimizing geodesic in the metric $g$ connecting $p$ and $q$ when $q \in \partial M$ as well, close enough to $p$; see also 19 . Let $\operatorname{If}(p, q)$ be the geodesic ray transform of the tensor field $f$ defined as an integral of $f_{i j} \dot{\gamma}^{i} \dot{\gamma}^{j}$ along $\gamma(p, q)$. All geodesics here are parameterized by a parameter in $[0,1]$ rather than being unit speed, and therefore the transform $I$ is parameterized differently than the usual one. Then $I(g-\tilde{g})>0$ by what we proved above. On the other hand,

$$
0<I(g-\tilde{g})=d^{2}(p, q)-I \tilde{g} \leq d^{2}(p, q)-\tilde{I} \tilde{g}=d^{2}(p, q)-\tilde{d}^{2}(p, q)=0
$$

because $\tilde{\gamma}(p, q)$ minimizes integrals of $g$ along curves connecting $p$ and $q$. This is a contradiction.

The starting point is an identity in 39. We will repeat the proof.

Let $V, \tilde{V}$ be two vector fields on a manifold $M$ (which will be replaced later with $\left.S^{*} M\right)$. Denote by $X\left(s, X^{(0)}\right)$ the solution of $\dot{X}=V(X), X(0)=X^{(0)}$, and we use the same notation for $\tilde{V}$ where the corresponding solutions are denoted by $\tilde{X}$. Then we have the following simple statement.

Lemma 2.2. For any $t>0$ and any initial condition $X^{(0)}$, if $\tilde{X}\left(\cdot, X^{(0)}\right)$ and $X\left(\cdot, X^{(0)}\right)$ exist on the interval $[0, t]$, then

$$
\begin{aligned}
& \tilde{X}\left(t, X^{(0)}\right)-X\left(t, X^{(0)}\right) \\
& \quad=\int_{0}^{t} \frac{\partial \tilde{X}}{\partial X^{(0)}}\left(t-s, X\left(s, X^{(0)}\right)\right)(\tilde{V}-V)\left(X\left(s, X^{(0)}\right)\right) d s .
\end{aligned}
$$

Proof. Set

$$
F(s)=\tilde{X}\left(t-s, X\left(s, X^{(0)}\right)\right) .
$$


Then

$$
\begin{aligned}
F^{\prime}(s)= & -\tilde{V}\left(\tilde{X}\left(t-s, X\left(s, X^{(0)}\right)\right)\right) \\
& +\frac{\partial \tilde{X}}{\partial X^{(0)}}\left(t-s, X\left(s, X^{(0)}\right)\right) V\left(X\left(s, X^{(0)}\right)\right) .
\end{aligned}
$$

The proof of the lemma would be complete by the fundamental theorem of calculus

$$
F(t)-F(0)=\int_{0}^{t} F^{\prime}(s) d s
$$

if we show the following

$$
\tilde{V}\left(\tilde{X}\left(t-s, X\left(s, X^{(0)}\right)\right)\right)=\frac{\partial \tilde{X}}{\partial X^{(0)}}\left(t-s, X\left(s, X^{(0)}\right)\right) \tilde{V}\left(X\left(s, X^{(0)}\right)\right) .
$$

Indeed, (2.3) follows from

$$
0=\left.\frac{d}{d \tau}\right|_{\tau=0} X(T-\tau, X(\tau, Z))=-V(X(T, Z))+\frac{\partial X}{\partial X^{(0)}}(T, Z) V(Z), \quad \forall T,
$$

after setting $T=t-s, Z=X\left(s, X^{(0)}\right)$.

Let $c, \tilde{c}$ be two speeds. Then the corresponding metrics are $g=c^{-2} d x^{2}$, and $\tilde{g}=\tilde{c}^{-2} d x^{2}$. The corresponding Hamiltonians and Hamiltonian vector fields are

$$
H=\frac{1}{2} c^{2} g_{0}^{i j} \xi_{i} \xi_{j}, \quad V=\left(c^{2} g_{0}^{-1} \xi,-\frac{1}{2} \partial_{x}\left(c^{2}|\xi|_{g_{0}}^{2}\right)\right)
$$

and the same ones related to $\tilde{c}$. We used the notation $|\xi|_{g_{0}}^{2}:=g_{0}^{i j} \xi_{i} \xi_{j}$.

We change the notation at this point. We denote points in the phase space $T^{*} M$, in a fixed coordinate system, by $z=(x, \xi)$. We denote the bicharacteristic with initial point $z$ by $Z(t, z)=(X(t, z), \Xi(t, z))$.

Then we get the identity already used in 39 .

$$
\tilde{Z}(t, z)-Z(t, z)=\int_{0}^{t} \frac{\partial \tilde{Z}}{\partial z}(t-s, Z(s, z))(\tilde{V}-V)(Z(s, z)) d s .
$$

We can naturally think of the scattering relation $L$ and the travel time $\ell$ as functions on the cotangent bundle instead of the tangent one. Then we get the following.

Proposition 2.1. Assume

$$
L\left(x_{0}, \xi^{0}\right)=\tilde{L}\left(x_{0}, \xi^{0}\right), \quad \ell\left(x_{0}, \xi^{0}\right)=\tilde{\ell}\left(x_{0}, \xi^{0}\right)
$$

for some $z_{0}=\left(x_{0}, \xi^{0}\right) \in \partial_{-} S^{*} M$. Then

$$
\int_{0}^{\ell\left(z_{0}\right)} \frac{\partial \tilde{Z}}{\partial z}\left(\ell\left(z_{0}\right)-s, Z\left(s, z_{0}\right)\right)(V-\tilde{V})\left(Z\left(s, z_{0}\right)\right) d s=0 .
$$


2.1. Linearization near $c=1$ and $g$ Euclidean. As a simple exercise, let $c=1$, $g_{i j}=\delta_{i j}$ and linearize for $\tilde{c}$ near 1 first under the assumption that $\tilde{c}=1$ outside an open region $\Omega \subset \mathbb{R}^{n}$. Then

$$
Z(s, z)=\left(\begin{array}{ll}
1 & s \\
0 & 1
\end{array}\right) z, \quad \frac{\partial Z(s, z)}{\partial z}=\left(\begin{array}{cc}
1 & s \\
0 & 1
\end{array}\right),
$$

and we get the following formal linearization of (2.4)

$$
\int\left(f \xi-\frac{1}{2}(t-s)\left(\partial_{x} f\right),-\frac{1}{2} \partial_{x} f\right)(x+s \xi, \xi) \mathrm{d} s=0,
$$

where

$$
f:=c^{2}-\tilde{c}^{2} .
$$

Notice that we would get the same thing if we replace $\partial \tilde{Z} / \partial z$ in (2.4) by $\partial Z / \partial z$. We integrate over the whole line $s \in \mathbb{R}$ because the integrand vanishes outside the interval $[0, \ell(x, \xi)]$. The last $n$ components of (2.7) imply

$$
\int \partial_{x} f(x+s \xi) \mathrm{d} s=0 .
$$

Now, assume that this holds for all $(x, \xi)$. Then $\partial_{x} f=0$, and since $f=0$ on $\partial M$, we get $f=0$.

2.2. The general case. We take the second $n$-dimensional component on (2.4) and use the fact that $c^{2}|\xi|_{g_{0}}^{2}=1$ on the bicharacteristics related to $c$. We assume that both geodesics extend to $t \in[0, \ell(x, \xi)]$. We want to emphasize that the bicharacteristics on the energy level $H=1 / 2$, related to $c$, do not necessarily stay on the same energy level for the Hamiltonian $\tilde{H}$. We get

$$
\begin{aligned}
& \int \frac{\partial \tilde{\Xi}}{\partial x}(\ell(z)-s, Z(s, z))\left(f g_{0}^{-1} \xi\right)(Z(s, z)) \mathrm{d} s \\
& -\frac{1}{2} \int \frac{\partial \tilde{\Xi}}{\partial \xi}(\ell(z)-s, Z(s, z))\left(\partial_{x}\left(f g_{0}^{-1}\right) \xi \cdot \xi\right)(Z(s, z)) \mathrm{d} s=0
\end{aligned}
$$

for any $z \in \partial_{-} S M$ for which (2.5) holds. As before, we integrate over $s \in \mathbb{R}$ because the support of the integrand vanishes for $s \notin[0, \ell(x, \xi)]$ (for that, we extend the bicharacteristics formally outside so that they do not come back). Write

$$
\partial_{x}\left(f g_{0}^{-1}\right)=g_{0}^{-1} \partial_{x} f+\left(\partial_{x} g_{0}^{-1}\right) f
$$

to get

$$
\begin{gathered}
0=\int \frac{\partial \tilde{\Xi}}{\partial x}(\ell(z)-s, Z(s, z))\left(f g_{0}^{-1} \xi\right)(Z(s, z)) \mathrm{d} s \\
-\frac{1}{2} \int \frac{\partial \tilde{\Xi}}{\partial \xi}(\ell(z)-s, Z(s, z))\left(\left(g_{0}^{-1} \partial_{x} f+f\left(\partial_{x} g_{0}^{-1}\right)\right) \xi \cdot \xi\right)(Z(s, z)) \mathrm{d} s .
\end{gathered}
$$

One of the terms on the right-hand side (r.h.s.) above involves $g_{0}^{-1} \xi \cdot \xi$ which equals $c^{-2}$ on the bicharacteristics of $H$ on the level $1 / 2$.

Introduce the exit times $\tau(x, \xi)$ defined as the minimal (and the only) $t>0$ so that $X(t, x, \xi) \in \partial M$. They are well defined near $S_{p} \partial M$, if $\partial M$ is strictly convex 
at $p$. We need to write $\frac{\partial \tilde{Z}}{\partial z}(\ell(z)-s, Z(s, z))$ as a function of $(x, \xi)=Z(s, z)$. We have

$$
\frac{\partial \tilde{Z}}{\partial z}(\ell(z)-s, Z(s, z))=\frac{\partial \tilde{Z}}{\partial z}(\tau(x, \xi),(x, \xi)) .
$$

Then we get, with $f$ as in (2.8),

$$
J_{i} f(\gamma):=\int\left(A_{i}^{j}(X(t), \Xi(t))\left(\partial_{x^{j}} f\right)(X(t))+B_{i}(X(t), \Xi(t)) f(X(t))\right) \mathrm{d} t=0
$$

for any bicharacteristic $\gamma=(X(t), \Xi(t))$ (related to the speed $c$ ) in our set, where

$$
\begin{aligned}
A_{i}^{j}(x, \xi)= & -\frac{1}{2} \frac{\partial \tilde{\Xi}_{i}}{\partial \xi_{j}}(\tau(x, \xi),(x, \xi)) c^{-2}(x), \\
B_{i}(x, \xi)= & \frac{\partial \tilde{\Xi}_{i}}{\partial x^{j}}(\tau(x, \xi),(x, \xi)) g_{0}^{i k}(x) \xi_{k} \\
& -\frac{1}{2} \frac{\partial \tilde{\Xi}_{i}}{\partial \xi_{j}}(\tau(x, \xi),(x, \xi))\left(\partial_{x^{j}} g_{0}^{-1}(x)\right) \xi \cdot \xi .
\end{aligned}
$$

A major inconvenience with this representation is that the exit time function $\tau(x, \xi)$ (recall that we assume strong convexity) becomes singular at $(x, \xi) \in T^{*} \partial M$. More precisely, the normal derivative w.r.t. $x$ when $\xi$ is tangent to $\partial M$ has a square root type of singularity. On the other hand, we have some freedom to extend the flow outside $M$ since we know that the jets of $c$ and $\tilde{c}$ at $\partial M$ are the same near $p$ : therefore, any smooth local extension of $c$ is also a smooth extension of $\tilde{c}$. Then for any $(x, \xi) \in \partial_{-} S^{*} M$ close enough to $S_{x_{0}}^{*} M$, the bicharacteristics originating from it will be identical once they exit $T^{*} M$ but are still close enough to it. Similarly, instead of starting from $T^{*} \partial M$, we could start at points and codirections close to it, but away from $\bar{M}$.

With this in mind, we push the boundary away a bit. Let $x_{0}$ represent the point $p$ near which we work, in a fixed coordinate system. Extend $g_{0}$ smoothly near $x_{0}$. Let $S\left(x_{0}, r\right)$ be the sphere in the metric $c^{-2} \mathrm{~d} x^{2}$ centered at $x_{0}$ with radius $0<r \ll 1$. For $(x, \xi)$ with $x$ in the geodesic ball $B\left(x_{0}, r\right)$, redefine $\tau(x, \xi)$ to be the travel time from $(x, \xi)$ to $S\left(x_{0}, r\right)$. Let $U_{-} \subset \partial_{-} S B\left(x_{0}, r\right)$ be the set of all points on $S\left(x_{0}, r\right)$ and incoming unit directions so that the corresponding geodesic in the metric $g$ is close enough to one tangent to $\partial M$ at $x_{0}$. Similarly, let $U_{+}$be the set of such pairs with outgoing directions. Redefine the scattering relation $L$ locally to act from $U_{-}$to $U_{+}$, and redefine $\ell$ similarly; see Figure 1, Then under the assumptions of Theorem 1.2. $L=\tilde{L}$ and $\ell=\tilde{\ell}$ on $U_{-}$. We can apply the construction above by replacing $\partial_{ \pm} S M$ locally by $U_{ \pm}$. The advantage we have now is that on $U_{-}$, the travel time $\tau$ is nonsingular. Equalities (2.12) and (2.13) are preserved then.

We now have

$$
\begin{aligned}
& A_{i}^{j}\left(x_{0}, \xi\right)=-\frac{1}{2} \frac{\partial \tilde{\Xi}_{i}}{\partial \xi_{j}}\left(r,\left(x_{0}, \xi\right)\right) c^{-2}\left(x_{0}\right) \\
& B_{i}\left(x_{0}, \xi\right)=\frac{\partial \tilde{\Xi}_{i}}{\partial x^{j}}\left(r,\left(x_{0}, \xi\right)\right) g_{0}^{i k}\left(x_{0}\right) \xi_{k}-\frac{1}{2} \frac{\partial \tilde{\Xi}_{i}}{\partial \xi_{j}}(r,(x, \xi))\left(\partial_{x^{j}} g_{0}^{-1}\left(x_{0}\right)\right) \xi \cdot \xi .
\end{aligned}
$$

Then by the strict convexity,

$$
A_{i}^{j}(x, \xi)=-\frac{1}{2} c^{-2}(x) \delta_{i}^{j}+O(r), \quad \text { for }(x, \xi) \in S^{*} M \text { near } S_{p}^{*} \partial M .
$$




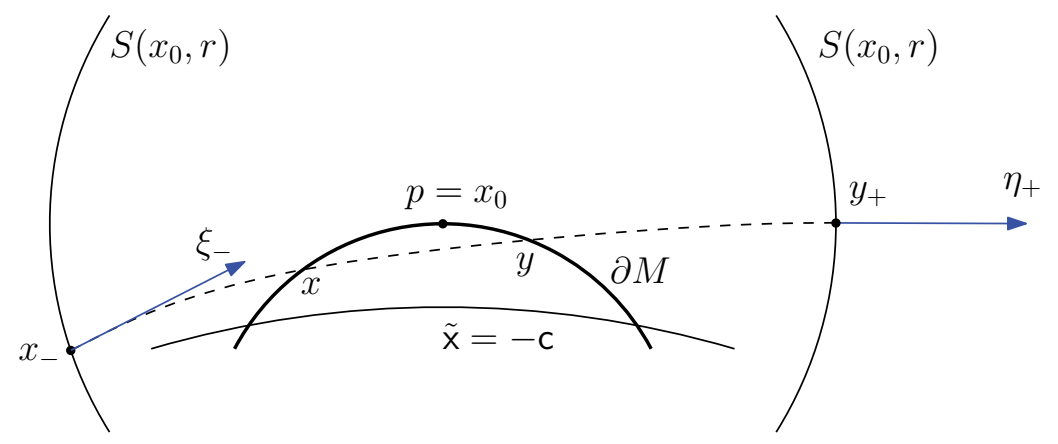

FigurE 1. The redefined scattering relation $\left(x_{-}, \xi_{-}\right) \mapsto\left(y_{+}, \eta_{+}\right)$.

2.3. A new linear problem. The arguments above lead to the following linear problem.

Problem. Assume (2.12) holds with some $f$ supported in $M$, for all geodesics close to the ones originating from $S_{x_{0}}^{*} \partial M$ (i.e., initial point $x_{0}$ and all unit initial codirections tangent to $\partial M)$. Assume that $\partial M$ is strictly convex at $x_{0}$ w.r.t. the speed $c$. Assume (2.15). Is it true that $f=0$ near $x_{0}$ ?

We show below in Proposition 3.3 that the answer is affirmative. Note that this reduces the original non-linear problem to a linear one, but this is not a linearization. Then Theorem 1.2 follows from it. On the other hand, Theorem 1.2 is not equivalent to that problem because the weight there has a specific structure, and thus Proposition 3.3 is a more general statement.

\section{LiNEAR ANALYSIS}

We first recall the setting introduced in [49] in our current notation. There the scalar x-ray transform along geodesics was considered, namely for $\beta \in S M$,

$$
(I f)(\beta)=\int_{\mathbb{R}} f\left(\gamma_{\beta}(t)\right) \mathrm{d} t
$$

where $\gamma_{\beta}$ is the geodesic with lift to $S M$ having starting point $\beta \in S M$. Here $M$ is assumed to have a strictly convex boundary, which can be phrased as the statement that if $\rho$ is a defining function for $\partial M$, then $-\left.\frac{\mathrm{d}^{2}}{\mathrm{~d} t^{2}}\left(\rho \circ \gamma_{\beta}\right)\right|_{t=0} \geq C>0$ whenever $\beta \in S \partial M$. One then considers a point $p \in \partial M$, and another function $\tilde{\mathrm{x}}$, denoted in 49 by $\tilde{x}$, such that $\tilde{\mathrm{x}}(p)=0, \mathrm{~d} \tilde{\mathrm{x}}(p)=-\mathrm{d} \rho(p)$, and the level sets of $\tilde{\mathrm{x}}$ near the 0 are strictly concave when viewed from the superlevel sets (which are on the side of $p$ when talking about the c-level set with $c<0)$, i.e., $\left.\frac{\mathrm{d}^{2}}{\mathrm{~d} t^{2}}\left(\tilde{\mathrm{x}} \circ \gamma_{\beta}\right)\right|_{t=0} \geq C>0$ if $\beta \in S\{\tilde{\mathrm{x}}=\mathrm{c}\}$, namely if $\left.\frac{\mathrm{d}}{\mathrm{d} t}\left(\tilde{\mathrm{x}} \circ \gamma_{\beta}\right)\right|_{t=0}=0$. For $\mathrm{c}>0$, we denote $M \cap\{\tilde{\mathrm{x}}>-\mathrm{c}\}$ by $\Omega_{c}$; we assume that $\mathrm{c}_{0}>0$ is such that $\overline{\Omega_{\mathrm{c}_{0}}}$ is compact on $M$, and the concavity assumption holds on $\Omega_{\mathrm{c}_{0}}$. Then it was shown that the x-ray transform $I$ restricted to $\beta \in S \overline{\Omega_{\mathrm{c}}}$ such that $\gamma_{\beta}$ leaves $\Omega_{\mathrm{c}}$ with both end points on $\partial M$ (i.e., at $\rho=0$ ) is injective if $\mathrm{c}>0$ is sufficiently small, and indeed one has a stability estimate for $f$ in terms of $I f$ on exponentially weighted spaces.

To explain this in detail, let $x=x_{c}=\tilde{x}+c$ be the boundary defining function of the artificial boundary, $\tilde{x}=-c$, that we introduced; indeed, it is convenient to work in $\tilde{M}$, a $C^{\infty}$ manifold extending $M$ across the boundary, extending $\tilde{x}$ to $\tilde{M}$ smoothly, 
and defining $\hat{\Omega}=\hat{\Omega}_{\mathrm{c}}=\left\{\mathrm{x}_{\mathrm{c}}>0\right\}$ as the extension of $\Omega$, so $\overline{\hat{\Omega}}$ is a smooth manifold with boundary, with only the artificial boundary being a boundary face. Then one writes $\beta=(\lambda, \omega)=\lambda \partial_{\mathrm{x}}+\omega \partial_{\mathrm{y}} \in S_{\mathrm{x}, \mathrm{y}} \tilde{M}$ relative to a product decomposition $\left(-\mathrm{c}_{0}, \mathrm{c}_{0}\right)_{\mathrm{x}} \times U_{\mathrm{y}}$ of $\tilde{M}$ near $p$. The concavity condition becomes that for $\beta$ whose $\lambda$-component vanishes,

$$
2 \alpha(\mathrm{x}, \mathrm{y}, 0, \omega)=\left.\frac{\mathrm{d}^{2}}{\mathrm{~d} t^{2}}\left(\mathrm{x} \circ \gamma_{\beta}\right)\right|_{t=0} \geq 2 C>0,
$$

with a new $C>0$; see the discussion preceding Equation (3.1) in [49]. For $\chi \in$ $C_{0}^{\infty}(\mathbb{R}), \chi \geq 0, \chi(0)>0$, one considers the map

$$
L_{0} v(\mathrm{x}, \mathrm{y})=\int_{\mathbb{R}} \int_{\mathbb{S}^{n-2}} \mathrm{x}^{-2} \chi(\lambda / \mathrm{x}) v(\mathrm{x}, \mathrm{y}, \lambda, \omega) \mathrm{d} \lambda \mathrm{d} \omega
$$

defined for $v$ a function on $S_{\overline{\hat{\Omega}}} \tilde{M}$. This differs from [49] in that the weight $\mathrm{x}^{-2}$ differs by 1 from the weight $\mathrm{x}^{-1}$ used in 49 ; this simply has the effect of removing an $\mathrm{x}^{-1}$ in [49, Proposition 3.3], as compared to the proposition stated below. If $\mathrm{c}$ is sufficiently small, or instead $\chi$ has sufficiently small support, for $(\mathrm{x}, \mathrm{y}) \in \bar{\Omega}, I$ only integrates over points in $\beta \in S \overline{\hat{\Omega}}$ such that $\gamma_{\beta}$ leaves $\Omega_{\mathrm{c}}$ with both end points on $\partial M$, i.e., over $\beta$ corresponding to $\Omega_{\mathrm{c}}$-local geodesics - the set of the latter is denoted by $\mathcal{M}_{\mathrm{c}}$. We refer to the discussion around [49, Equation (3.1)] for more detail, but roughly speaking the concavity of the level sets of $x$ means that the geodesics that are close to being tangent to the foliation, with "close" measured by the distance from the artificial boundary, $x=0$, then they cannot reach $x=0$ (or reach again, in case they start there) without reaching $x=c^{\prime}$ for some fixed $c^{\prime}>0$; notice that the geodesics involved in the integration through a point on the level set $x=\tilde{c}$ make an angle $\lesssim \tilde{c}$ with the tangent space of the level set due to the compact support of $\chi$. Then we consider the map $P=L_{0} \circ I$. The main technical result of [49, whose notation involving the so-called scattering Sobolev spaces $H_{\mathrm{sc}}^{s, r}(\overline{\hat{\Omega}})$ and scattering pseudo-differential operators $\Psi_{\mathrm{sc}}^{s, r}(\overline{\hat{\Omega}})$ is explained below, was the following proposition.

Proposition 3.1 (See [49, Proposition 3.3 and Lemma 3.6]). For $\digamma>0$ let

$$
P_{\digamma}=e^{-\digamma / x} P e^{\digamma / x}: C_{0}^{\infty}(\overline{\hat{\Omega}}) \rightarrow C^{\infty}(\overline{\hat{\Omega}}) .
$$

Then $P_{\digamma} \in \Psi_{\mathrm{sc}}^{-1,0}(\overline{\hat{\Omega}})$.

Further, if c $>0$ is sufficiently small, then for a suitable choice of $\chi \in C_{0}^{\infty}(\mathbb{R})$ with $\chi(0)=1, \chi \geq 0, P$ is elliptic in $\Psi_{\mathrm{sc}}^{-1,0}(\overline{\hat{\Omega}})$ on a neighborhood of $\bar{\Omega}$.

Shrinking c further if needed, $P_{\digamma}$ satisfies the estimate

$$
\|v\|_{H_{\mathrm{sc}}^{s, r}(\overline{\hat{\Omega}})} \leq C\left\|P_{\digamma} v\right\|_{H_{\mathrm{sc}}^{s+1, r}(\overline{\hat{\Omega}})}
$$

for $v$ supported in $M \cap \overline{\hat{\Omega}}$.

We now briefly explain the role of the so-called scattering pseudo-differential operators and the corresponding Sobolev spaces (which are typically used to study phenomena "at infinity") in our problem (where there is no obvious "infinity"); we refer to [49, Section 2] for a more thorough exposition. These concepts were introduced by Melrose 20, in a general geometric setting, but on $\mathbb{R}^{n}$ these operators actually correspond to a special case of Hörmander's Weyl calculus [15, also 
studied earlier by Šubin [37] and Parenti [25]. So consider the reciprocal spherical coordinate map, $(0, \epsilon)_{\times} \times \mathbb{S}_{\theta}^{n-1} \rightarrow \mathbb{R}^{n}$, with $\mathbb{S}^{n-1} \subset \mathbb{R}^{n}$ (unit sphere), and map $(\mathrm{x}, \theta) \mapsto \mathrm{x}^{-1} \theta \in \mathbb{R}^{n}$. This map is a diffeomorphism onto its range, and it provides a compactification of $\mathbb{R}^{n}$ (the so-called radial or geodesic compactification) by adding $\{0\}_{x} \times \mathbb{S}_{\theta}^{n-1}$ as infinity to $\mathbb{R}^{n}$, to obtain $\overline{\mathbb{R}^{n}}$, which is now diffeomorphic to a ball. Now for general $U_{\mathrm{y}}$ above, we may regard, at least locally ${ }^{3} U_{\mathrm{y}}$ also as a coordinate chart in $\mathbb{S}^{n-1}$, and thus obtain an identification of $\bar{\Omega}$ with a region intersection $\overline{\mathbb{R}^{n}}$; thus our artificial boundary $\mathrm{x}=0$ corresponds to infinity at $\mathbb{R}^{n}$. In particular, notions from $\mathbb{R}^{n}$ can now be transferred to a neighborhood of our artificial boundary. Since the relevant vector fields on $\mathbb{R}^{n}$ are generated by translation invariant vector fields, which are complete under the exponential map, the transferred analysis replaces the incomplete geometry of standard vector fields on $\overline{\hat{\Omega}}$ by a complete one. Concretely, these vector fields, when transferred, become linear combinations of $x^{2} \partial_{x}$ and $x \partial_{y_{j}}$, with smooth coefficients. In particular, these are the vector fields with respect to which Sobolev regularity is measured. Thus, $H_{\mathrm{sc}}^{s, r}(\overline{\hat{\Omega}})$ is the so-called scattering Sobolev space, which is locally, under the above identification, just the standard weighted Sobolev space $H^{s, r}\left(\mathbb{R}^{n}\right)$ (see [49, Section 2]), while $\Psi_{\text {sc }}^{s, r}(\overline{\hat{\Omega}})$ is Melrose's scattering pseudo-differential algebra, which locally, again under this identification, simply corresponds to quantizing symbols $a$ with $\left|D_{z}^{\alpha} D_{\zeta}^{\beta} a\right| \leq C\langle z\rangle^{r-|\alpha|}\langle\zeta\rangle^{s-|\beta|}$ on $T^{*} \mathbb{R}^{n}=\mathbb{R}_{z}^{n} \times \mathbb{R}_{\zeta}^{n}$ (see again [49, Section 2] for more detail). Note that ellipticity in this algebra, called full ellipticity, is in the sense as both $|z| \rightarrow \infty$ and $|\zeta| \rightarrow \infty$, i.e., modulo symbols one order lower and with an extra order of decay as $|z| \rightarrow \infty$.

Notice that (3.1) implies the estimate

$$
\|f\|_{e^{\digamma / \times} H_{\mathrm{sc}}^{s, r}(\bar{\Omega})} \leq C\|P f\|_{e^{\digamma / \times} H_{\mathrm{sc}}^{s+1, r}(\bar{\Omega})}
$$

for the unconjugated operator, valid when $f$ is supported in $M \cap \overline{\hat{\Omega}}$. Rewriting $P$ as $L_{0} \circ I$, this gives that for $\delta>0, s \geq-1$,

$$
\|f\|_{e^{(\digamma+\delta) / \times} H^{s}(\bar{\Omega})} \leq C\|I f\|_{e^{\digamma / \times} H^{s+1}\left(\mathcal{M}_{c}\right)} ;
$$

see the discussion in [4] after Lemma 3.6.

After this recollection, we continue by generalizing (2.12) to regard the functions $\partial_{x_{j}} f$ and $f$ entering into it as independent unknowns, while restricting the transform to the region of interest $\Omega=\Omega_{\mathrm{c}}$. So let $\tilde{J}_{i}$ be defined by

$$
\begin{aligned}
& \tilde{J}_{i}\left(u_{0}, u_{1}, \ldots, u_{n}\right)(\beta) \\
& \quad:=\int_{\gamma_{\beta}}\left(A_{i}^{j}(X(t), \Xi(t)) u_{j}(X(t))+B_{i}(X(t), \Xi(t)) u_{0}(X(t))\right) \mathrm{d} t,
\end{aligned}
$$

where $\gamma_{\beta}$ is the geodesic with lift to $S \Omega$ having starting point $\beta \in S \Omega$. Let $\tilde{J}=\left(\tilde{J}_{1}, \ldots, \tilde{J}_{n}\right)$. This is a vector valued version of the geodesic x-ray transform considered in [49], and described above, sending functions on $\Omega$ with values in

\footnotetext{
${ }^{3}$ That is, possibly at the cost of shrinking it; in fact, all concepts below are essentially local within $\mathbb{S}^{n-1}$; thus even in full generality one can reduce scattering objects to (conic regions near infinity in) $\mathbb{R}^{n}$ this way, much as standard Sobolev spaces and pseudo-differential operators are so reducible to subsets of $\mathbb{R}^{n}$ with compact closure.
} 
$\mathbb{C}^{n+1}$ to functions with values in $\mathbb{C}^{n}$. We then define $L$ as a map from $\mathbb{C}^{n}$-valued functions on $S \Omega$ to $\mathbb{C}^{n}$ valued functions on $\Omega$ by

$$
L v(\mathrm{x}, \mathrm{y})=\int_{\mathbb{R}} \int_{\mathbb{S}^{n-2}} \mathrm{x}^{-2} \chi(\lambda / \mathrm{x}) v(\mathrm{x}, \mathrm{y}, \lambda, \omega) \mathrm{d} \lambda \mathrm{d} \omega
$$

as in [49]; this is a diagonal operator: $L=L_{0} \otimes \mathrm{Id}$. Then we consider the map $P=L \circ \tilde{J}$, and in addition to the properties mentioned above in the scalar setting, we are also interested in continuity properties in terms of the background data, such as in the background metric as well as the function $x$. Recall that the map

$$
\Gamma_{+}: S \tilde{M} \times[0, \infty) \rightarrow[\tilde{M} \times \tilde{M} ; \operatorname{diag}], \Gamma_{+}(\mathrm{x}, \mathrm{y}, \lambda, \omega, t)=\left((\mathrm{x}, \mathrm{y}), \gamma_{\mathrm{x}, \mathrm{y}, \lambda, \omega}(t)\right)
$$

is a local diffeomorphism, and similarly for $\Gamma_{-}$in which $(-\infty, 0]$ takes the place of $[0, \infty)$ (see the discussion around [49, Equations (3.2) and (3.3)]); indeed, this is true for more general curve families. Here $[\tilde{M} \times \tilde{M}$; diag] is the blowup of $\tilde{M}$ at the diagonal $z=z^{\prime}$, which essentially means the introduction of spherical/polar coordinates, or often more conveniently projective coordinates, about it. Concretely, writing the (local) coordinates from the two factors of $\tilde{M}$ as $\left(z, z^{\prime}\right)$,

$$
z,\left|z-z^{\prime}\right|, \frac{z-z^{\prime}}{\left|z-z^{\prime}\right|}
$$

give (local) coordinates on this space. Note that when $\Gamma_{ \pm}$are given by geodesics of a metric $g$ just $C^{1}$-near a fixed background metric $g_{0}$, as $C^{\infty}$ maps, $\Gamma_{ \pm}$depend continuously on $g$ in the $C^{\infty}$ topology.

In order to consider continuity properties in $\tilde{x}$ in a $C^{1}$-neighborhood of a fixed function $\tilde{\mathrm{x}}_{0}$, it is convenient to use the map $(\tilde{\mathrm{x}}, \mathrm{y})$ to identify a neighborhood of $p$ with a neighborhood $O$ of the origin in $\mathbb{R} \times U$. Thus, for c fixed, but $\tilde{\mathrm{x}}$ being $C^{1}$-close to $\tilde{\mathrm{x}}_{0}$, on this fixed background $O \subset \mathbb{R} \times U$, the pulled back metrics $\underline{g}$ depend continuously on $(\tilde{\mathrm{x}}, g)$ as maps $C^{\infty}(\tilde{M}) \times C^{\infty}\left(\tilde{M} ; S^{2} \tilde{M}\right) \rightarrow C^{\infty}(O)$; this normalizes $\tilde{\mathrm{x}}$ to be simply the first coordinate function $\underline{\tilde{x}}$ on $O$. Correspondingly, below, the continuous dependence, of all objects discussed, on $\tilde{x}$ (in the $C^{\infty}$ topology) is automatic: what is meant always is that by pullback to $O$ the resulting objects, living on fixed domains such as $\{\underline{\tilde{x}}+c>0\}$, depend continuously on $g$ and $\tilde{x}$, which follows from the continuous dependence of these objects on $g$. Since we do not want to overburden the notation, we do not write this pullback explicitly.

The main technical result here is the following proposition.

Proposition 3.2. For $\digamma>0$, let

$$
P_{\digamma}=e^{-\digamma / x} P e^{\digamma / x}: C_{0}^{\infty}\left(\overline{\hat{\Omega}} ; \mathbb{C}^{n+1}\right) \rightarrow C^{\infty}\left(\overline{\hat{\Omega}} ; \mathbb{C}^{n}\right) .
$$

Then $P_{\digamma} \in \Psi_{\mathrm{sc}}^{-1,0}\left(\overline{\hat{\Omega}} ; \mathbb{C}^{n+1}, \mathbb{C}^{n}\right)$, and the map $\Gamma_{ \pm} \mapsto P_{\digamma}$ is continuous from the $C^{\infty}$ topology to the Fréchet topology of $\Psi_{\mathrm{sc}}^{-1,0}\left(\overline{\hat{\Omega}} ; \mathbb{C}^{n+1}, \mathbb{C}^{n}\right)$.

Further, if $\mathrm{c}$ is sufficiently small and (2.15) holds, then for a suitable choice of $\chi \in C_{0}^{\infty}(\mathbb{R})$ with $\chi(0)=1, \chi \geq 0$, if we write $P_{\digamma}=\left(P_{0}, \tilde{P}\right)$, with $P_{0}$ corresponding to the first component, $\tilde{P}$ the last $n$ components, in the domain space, then $\tilde{P} i s$ elliptic in $\Psi_{\mathrm{sc}}^{-1,0}\left(\bar{\Omega} ; \mathbb{C}^{n}, \mathbb{C}^{n}\right)$ in a neighborhood of $\bar{\Omega}$.

Remark 3.1. Notice that ellipticity being an open condition, this means that there exists $\mathrm{c}_{0}>0$ such that if $\mathrm{c}<\mathrm{c}_{0}$, then the same $\chi$ works for all $g C^{\infty}$-close to $g_{0}$. 
Further, in view of the paragraph preceding the proposition, the map $\left(\tilde{\mathrm{x}}, \Gamma_{ \pm}\right) \mapsto$ $P_{\digamma}$ is continuous from the $C^{\infty}$ topology to the Fréchet topology of $\Psi_{\mathrm{sc}}^{-1,0}\left(\overline{\hat{\Omega}} ; \mathbb{C}^{n+1}\right.$, $\left.\mathbb{C}^{n}\right)$, where the latter is understood to actually stand for $\Psi_{\mathrm{sc}}^{-1,0}\left(\{\underline{\tilde{x}}+\mathrm{c} \geq 0\} ; \mathbb{C}^{n+1}, \mathbb{C}^{n}\right)$ via the identifications discussed above.

Proof. This is simply a vector valued version of [49, Proposition 3.3] and [49, Lemma 3.6], recalled above in Proposition 3.1, In particular, to show $P \in \Psi_{\mathrm{sc}}^{-1,0}(\bar{\Omega}$; $\left.\mathbb{C}^{n+1}, \mathbb{C}^{n}\right)$, it suffices to show that $P$ is a matrix of pseudo-differential operators $P_{i j} \in \Psi_{\mathrm{sc}}^{-1,0}(\hat{\Omega}), i=1,2, \ldots, n, j=0,1,2, \ldots, n$, depending continuously on $\Gamma_{ \pm}$. But for $j>0$, with $j=0$ being completely analogous, $P_{i j} w$ has the form

$$
\int_{\mathbb{R}} \int_{\mathbb{S}^{n-2}} \mathrm{x}^{-2} \chi(\lambda / \mathrm{x}) \int A_{i}^{j}\left(X_{\mathrm{x}, \mathrm{y}, \lambda, \omega}(t), \Xi_{\mathrm{x}, \mathrm{y}, \lambda, \omega}(t)\right) w\left(X_{\mathrm{x}, \mathrm{y}, \lambda, \omega}(t)\right) \mathrm{d} t \mathrm{~d} \lambda \mathrm{d} \omega .
$$

The only difference from [49, Proposition 3.3] then is the presence of the weight factor

$$
A_{i}^{j}\left(X_{x, y, \lambda, \omega}(t), \Xi_{x, y, \lambda, \omega}(t)\right)
$$

It is convenient to rewrite this via the metric identification, say by $g_{0}$, in terms of tangent vectors. Changing the notation for the flow, in our coordinates $(x, y, \lambda, \omega)$, writing now

$$
\left(\gamma_{x, y, \lambda, \omega}(t), \gamma_{x, y, \lambda, \omega}^{\prime}(t)\right)=\left(\mathbf{X}_{x, y, \lambda, \omega}(t), \mathbf{Y}_{x, y, \lambda, \omega}(t), \Lambda_{x, y, \lambda, \omega}(t), \Omega_{x, y, \lambda, \omega}(t)\right)
$$

for the lifted geodesic $\gamma_{x, y, \lambda, \omega}(t)$,

$$
\tilde{A}_{i}^{j}\left(\mathrm{X}_{\mathrm{x}, \mathrm{y}, \lambda, \omega}(t), \mathrm{Y}_{\mathrm{x}, \mathrm{y}, \lambda, \omega}(t), \Lambda_{\mathrm{x}, \mathrm{y}, \lambda, \omega}(t), \Omega_{\mathrm{x}, \mathrm{y}, \lambda, \omega}(t)\right)
$$

replaces $A_{i}^{j}\left(X_{\mathrm{x}, \mathrm{y}, \lambda, \omega}(t), \Xi_{\mathrm{x}, \mathrm{y}, \lambda, \omega}(t)\right)$. As in [49, Proposition 3.3] one rewrites the integral in terms of coordinates $\left(\mathrm{x}, \mathrm{y}, \mathrm{x}^{\prime}, \mathrm{y}^{\prime}\right)$ on the left and right factors of $\overline{\hat{\Omega}}$ (i.e., one explicitly expresses the Schwartz kernel), using that the map $\Gamma_{+}$of (3.2) is a local diffeomorphism, and similarly for $\Gamma_{-}$; we again refer to the discussion around [49, Equations (3.2) and (3.3)]. Further,

$$
(\mathrm{x}, \mathrm{y}, \lambda, \omega, t) \mapsto \gamma_{\mathrm{x}, \mathrm{y}, \lambda, \omega}^{\prime}(t)
$$

is a smooth map $S M \times \mathbb{R} \rightarrow \mathbb{R}^{n}$ (depending continuously on $\Gamma_{ \pm}$in the respective $C^{\infty}$ topologies) so composing it with $\Gamma_{ \pm}^{-1}$ from the right, one can re-express the integral giving $P_{i j} w$ away from the boundary as

$$
\int w\left(z^{\prime}\right)\left|z-z^{\prime}\right|^{-n+1} b\left(z, \frac{z-z^{\prime}}{\left|z-z^{\prime}\right|},\left|z-z^{\prime}\right|\right) \mathrm{d} z^{\prime}
$$

as in [49, Equation (3.7)], with $b$ a smooth function of the indicated variables (thus smooth on $[\tilde{M} \times \tilde{M} ; \operatorname{diag}])$, depending continuously on $\Gamma_{ \pm}$in the respective $C^{\infty}$ topologies, and with

$$
b\left(z, \frac{z-z^{\prime}}{\left|z-z^{\prime}\right|}, 0\right)=\tilde{\chi}\left(z, \frac{z^{\prime}-z}{\left|z^{\prime}-z\right|}\right) \tilde{A}_{i}^{j}\left(z, \frac{z^{\prime}-z}{\left|z^{\prime}-z\right|}\right) \sigma\left(z, \frac{z^{\prime}-z}{\left|z^{\prime}-z\right|}\right)
$$

with $\sigma>0$, bounded below by a positive constant, by a weight factor, and where $\chi(\lambda / \mathrm{x})$ is written as $\tilde{\chi}(\mathrm{x}, \mathrm{y}, \lambda, \omega)$. Recall from [49, Section 2] that coordinates on Melrose's scattering double space, on which the Schwartz kernels of elements of $\Psi_{\mathrm{sc}}^{s, r}(\overline{\hat{\Omega}})$ are conormal to the diagonal, near the lifted scattering diagonal, are (with $\mathrm{x} \geq 0$ )

$$
\mathrm{x}, \mathrm{y}, X=\frac{\mathrm{x}-\mathrm{x}^{\prime}}{\mathrm{x}^{2}}, Y=\frac{\mathrm{y}-\mathrm{y}^{\prime}}{\mathrm{x}}
$$


Further, it is convenient to write coordinates on $[\tilde{M} \times \tilde{M} ; \operatorname{diag}]$ in the region of interest (see the beginning of the paragraph of Equation (3.10) in [49]), namely (the lift of) $\left|\mathrm{x}-\mathrm{x}^{\prime}\right|<C\left|\mathrm{y}-\mathrm{y}^{\prime}\right|$, as

$$
x, y,\left|y-y^{\prime}\right|, \frac{x-x^{\prime}}{\left|y-y^{\prime}\right|}, \frac{y-y^{\prime}}{\left|y-y^{\prime}\right|},
$$

with the norms being Euclidean norms 4 instead of (3.3); we write $\Gamma_{ \pm}$in terms of these. Note that these are $\mathrm{x}, \mathrm{y}, \mathrm{x}|Y|, \frac{\mathrm{x}|X|}{|Y|}, \hat{Y}$. Then, similarly, near the boundary as in [49, Equation (3.13)], one obtains the Schwartz kernel

$$
\begin{aligned}
& K^{\mathrm{b}}(\mathrm{x}, \mathrm{y}, X, Y) \\
& =\sum_{ \pm} e^{-\digamma X /(1+\mathrm{x} X)} \chi\left(\frac{X}{|Y|}+|Y| \tilde{\Lambda}_{ \pm}\left(\mathrm{x}, \mathrm{y}, \mathrm{x}|Y|, \frac{\mathrm{x}|X|}{|Y|}, \hat{Y}\right)\right) \\
& \quad \times \tilde{A}_{i}^{j}\left(\Gamma_{ \pm}^{-1}\left(\mathrm{x}, \mathrm{y}, \mathrm{x}|Y|, \frac{\mathrm{x} X}{|Y|}, \hat{Y}\right)\right)|Y|^{-n+1} J_{ \pm}\left(\mathrm{x}, \mathrm{y}, \frac{X}{|Y|},|Y|, \hat{Y}\right),
\end{aligned}
$$

with the density factor $J$ smooth, positive, depending continuously on $\Gamma_{ \pm}$in the respective $C^{\infty}$ topologies, $=1$ at $\mathrm{x}=0$. Here

$$
\mathrm{x}, \mathrm{y},|Y|, \frac{X}{|Y|}, \hat{Y}
$$

are valid coordinates on the blowup of the scattering diagonal in $|Y|>\epsilon|X|, \epsilon>0$, which is the case automatically on the support of the kernel due to the argument of $\chi$; cf. the discussion after [49, Equation (3.12)], so the argument of $\tilde{A}_{i}^{j} \circ \Gamma_{ \pm}^{-1}$ is smooth on this blown-up space still depending continuously on $\Gamma_{ \pm}$in the respective $C^{\infty}$ topologies. We can evaluate this argument: for instance, by [49, Equation (3.10)],

$$
\left(\Lambda \circ \Gamma_{ \pm}^{-1}\right)\left(\mathrm{x}, \mathrm{y}, \mathrm{x}|Y|, \frac{\mathrm{x} X}{|Y|}, \hat{Y}\right)=\mathrm{x} \frac{X}{|Y|}+\mathrm{x}|Y| \tilde{\Lambda}_{ \pm}\left(\mathrm{x}, \mathrm{y}, \mathrm{x}|Y|, \frac{\mathrm{x} X}{|Y|}, \hat{Y}\right)
$$

with $\tilde{\Lambda}_{ \pm}$smooth, while the subsequent equation in the same location gives

$$
\left(\Omega \circ \Gamma_{ \pm}^{-1}\right)\left(\mathrm{x}, \mathrm{y}, \mathrm{x}|Y|, \frac{\mathrm{x} X}{|Y|}, \hat{Y}\right)=\hat{Y}+\mathrm{x}|Y| \tilde{\Omega}_{ \pm}\left(\mathrm{x}, \mathrm{y}, \mathrm{x}|Y|, \frac{\mathrm{x} X}{|Y|}, \hat{Y}\right)
$$

with $\tilde{\Omega}_{ \pm}$smooth; here, both $\tilde{\Lambda}_{ \pm}$and $\tilde{\Omega}_{ \pm}$depend continuously on $\Gamma_{ \pm}$in the respective $C^{\infty}$ topologies. This proves the first part of the proposition as in 49, Proposition 3.3].

To prove the second part, note that in view of (3.4) (which just needs to be evaluated at $\mathrm{x}=0$ ), 49, Lemma 3.5] is replaced by the statement that the boundary principal symbol of $P_{i j}$ in $\Psi_{\mathrm{sc}}^{-1,0}(\overline{\hat{\Omega}})$ is twice the $(X, Y)$-Fourier transform of

$$
e^{-\digamma X} \chi\left(\frac{X-\alpha(0, \mathrm{y}, 0, \hat{Y})|Y|^{2}}{|Y|}\right) \tilde{A}_{i}^{j}(0, \mathrm{y}, 0,0, \hat{Y})|Y|^{-n+1},
$$

while for $P_{i 0}$ it is twice the $(X, Y)$-Fourier transform of

$$
e^{-\digamma X} \chi\left(\frac{X-\alpha(0, \mathrm{y}, 0, \hat{Y})|Y|^{2}}{|Y|}\right) \tilde{B}_{i}(0, \mathrm{y}, 0,0, \hat{Y})|Y|^{-n+1},
$$

\footnotetext{
${ }^{4}$ This is an example of partial projective coordinates for a blowup.

${ }^{5}$ This is another example of partial projective coordinates for a blowup.
} 
with $\tilde{B}_{i}$ defined analogously to $\tilde{A}_{i}^{j}$. (Recall that $2 \alpha(\mathrm{x}, \mathrm{y}, \lambda, \omega)$ is the $\mathrm{x}$ component of $\gamma_{x, y, \lambda, \omega}^{\prime \prime}(0)$, and the convexity assumption on $\mathrm{x}$ is that $\alpha$ is positive; see [49] above Equation (3.1).) For $A_{i}^{j}=-\frac{1}{2} c^{-2}\left(x_{0}\right) \delta_{i}^{j}$ (see (2.15)), the invertibility of the principal symbol, with values in $n \times n$ matrices, of the principal symbol of $\tilde{P}$ follows when $\chi$ is chosen as in [49, Lemma 3.6], for it is $-\frac{1}{2} c^{-2}\left(x_{0}\right)$ times the boundary symbol in [49, Lemma 3.6] times the $n \times n$ identity matrix. In general, due to the perturbation stability of the property of invertibility, the same follows for $c$ sufficiently small.

Corollary 3.1. With the notation of Proposition 3.2 there is $\tilde{c}>0$ such that if $0<\mathrm{c}<\tilde{\mathrm{c}}$, then $P_{\digamma}$ satisfies the estimate

$$
\|u\|_{H_{\mathrm{sc}}^{s, r}(\overline{\hat{\Omega}})} \leq C\left\|P_{\digamma} u\right\|_{H_{\mathrm{sc}}^{s+1, r}(\overline{\hat{\Omega}})}+C\left\|u_{0}\right\|_{H_{\mathrm{sc}}^{s, r}(\overline{\hat{\Omega}})}
$$

for $u$ supported in $M \cap \overline{\hat{\Omega}}$, with the constant $C$ uniform in c. Further, fixing $s, r$, there exist $k, \epsilon>0$, and $\rho_{0}<0$ such that $C$ can be taken uniform for $\Gamma_{ \pm} \epsilon$-close to a reference $\Gamma_{ \pm}^{0}$ in $C^{k}$, and the estimate holds even for $u$ supported in $\rho \geq \rho_{0}$ (in place of $\rho \geq 0$ ).

Remark 3.2. As in the case of the preceding proposition, the dependence on $\tilde{x}$ is also continuous; i.e., by possibly increasing $k, C$ can be taken uniform for $\Gamma_{ \pm} C^{k}$-close to a reference $\Gamma_{ \pm}^{0}$ and $\tilde{x} C^{k}$-close to a reference $\tilde{x}_{0}$.

Proof. By the density of elements of $\dot{C}^{\infty}(M \cap \overline{\hat{\Omega}})$ in $H_{\mathrm{sc}}^{s, r}(\overline{\hat{\Omega}})$ supported in $M \cap \overline{\hat{\Omega}}$, it suffices to consider $u \in \dot{C}^{\infty}(M \cap \overline{\hat{\Omega}})$ to prove (3.6).

Consider $s=0, r=0$. Let $\Lambda \in \Psi_{\mathrm{sc}}^{1,0}(\hat{\Omega})$ be elliptic and invertible (one can, e.g., locally identify $\Omega$ with $\mathbb{R}^{n}$; then on the Fourier transform side multiplication by $\langle\xi\rangle$ works). Thus, $\Lambda P_{\digamma}$, with $\Lambda$ acting diagonally on $\dot{C}^{\infty}\left(\overline{\hat{\Omega}} ; \mathbb{C}^{n}\right)$, is in $\Psi_{\text {sc }}^{0,0}\left(\overline{\hat{\Omega}} ; \mathbb{C}^{n+1}, \mathbb{C}^{n}\right)$, depending continuously, in the Fréchet topology of pseudodifferential operators, on $\Gamma_{ \pm}$in $C^{\infty}$, and $\Lambda \tilde{P} \in \Psi_{\mathrm{sc}}^{0,0}\left(\overline{\hat{\Omega}} ; \mathbb{C}^{n}, \mathbb{C}^{n}\right)$ is elliptic in $\Psi_{\mathrm{sc}}^{0,0}(\overline{\hat{\Omega}} ;$ $\left.\mathbb{C}^{n}, \mathbb{C}^{n}\right)$ locally in a neighborhood of $\Omega$. This implies, as presented in [49] after Lemma 3.6 (without the uniform discussion on $\Gamma_{ \pm}$), relying on the arguments at the end of Section 2 there, that there exist $k$ and $\epsilon>0$ such that if $\Gamma_{ \pm}$is $\epsilon$-close to $\Gamma_{ \pm}^{0}$ in $C^{k}$, then, if c $>0$ is sufficiently small, $\Lambda \tilde{P}$ satisfies

$$
\|w\|_{L_{\mathrm{sc}}^{2}(\overline{\hat{\Omega}})} \leq C_{0}\|\Lambda \tilde{P} w\|_{L_{\mathrm{sc}}^{2}(\bar{\Omega})}
$$

for $w$ supported in $\bar{\Omega}$. Here $L_{\mathrm{sc}}^{2}(\overline{\hat{\Omega}})=H_{\mathrm{sc}}^{0,0}(\overline{\hat{\Omega}})$ is the $L^{2}$ space relative to a nondegenerate scattering density - the latter are equivalent to the lifted Lebesgue measure from $\mathbb{R}^{n}$, and thus are bounded multiples of $\frac{d x d y}{x^{n+1}}$.

We recall the essential part of this argument briefly. One considers the whole family of domains $\overline{\hat{\Omega}}_{\mathrm{c}}$, which can be identified with each other locally in the region of interest by the maps $\Phi_{c}(\tilde{x}, y)=(\tilde{x}+c, y)$, i.e., simply translation in the $\tilde{x}$-coordinate, so instead of considering a family of spaces with an operator on each of them, one can consider a fixed space, denote this by $\overline{\hat{\Omega}}_{0}$, with a continuous (in c) family of operators, $T_{\mathrm{c}}$, namely $T_{\mathrm{c}}=\left(\Phi_{\mathrm{c}}^{-1}\right)^{*} \Lambda \tilde{P} \Phi_{\mathrm{c}}^{*}$; these depend continuously on $\Gamma_{ \pm}$in the Fréchet sense discussed above. Notice that we are interested in the 
region $6 \Omega_{c}$, and that there is a continuous function $f$ on $\mathbb{R}$ with $f(0)=0$ and $\mathrm{x}_{\mathrm{c}} \leq f(\mathrm{c})$ on $\Omega_{\mathrm{c}}$. Correspondingly, in the translated space, $\tilde{\mathrm{x}} \leq f(\mathrm{c})$ on the image of $\Omega_{c}$; notice that this region shrinks as $\mathrm{c}>0$ goes to 0 . On the other hand, there is a fixed open set $O \subset \overline{\hat{\Omega}}$, a neighborhood of $x_{0}$, on which the operators $T_{\mathrm{c}}$ are elliptic in $\Psi_{\text {sc }}^{0,0}(\overline{\hat{\Omega}})$ for $0 \leq|\mathrm{c}|<\mathrm{c}_{0}$. Let $K_{0}$ be a compact subset of $O$, still including a neighborhood of $x_{0}, \phi \in C_{0}^{\infty}(O)$ be identically 1 on a neighborhood of $K$. Then the elliptic parametrix construction (which is local, and uniform in c by the continuity) produces a parametrix family $G_{\mathrm{c}} \in \Psi_{\mathrm{sc}}^{0,0}\left(\overline{\hat{\Omega}} ; \mathbb{C}^{n}, \mathbb{C}^{n}\right), G_{\mathrm{c}} T_{\mathrm{c}}=\mathrm{Id}+E_{\mathrm{c}}$, where $E_{\mathrm{c}} \in \Psi_{\mathrm{sc}}^{0,0}\left(\overline{\hat{\Omega}} ; \mathbb{C}^{n}, \mathbb{C}^{n}\right)$ only, but $\phi E_{\mathrm{c}} \phi \Psi_{\mathrm{sc}}^{-\infty,-\infty}\left(\overline{\hat{\Omega}} ; \mathbb{C}^{n}, \mathbb{C}^{n}\right)$, uniformly in c, depending continuously on $\Gamma_{ \pm}$in the Fréchet topologies. Then (multiplying the parametrix identity by $\phi$ from left and right and applying to $v$ ) for $v$ supported in $K,\left(\mathrm{Id}+\phi E_{\mathrm{c}} \phi\right) v=\phi G_{\mathrm{c}} T_{\mathrm{c}} v$. Now, the Schwartz kernel of $\phi E_{\mathrm{c}} \phi$ is Schwartz, i.e., is bounded by $C_{N}\left(\mathrm{xx}^{\prime}\right)^{N}$ for any $N$, uniformly in c. (Here we write, say, the Schwartz kernel relative to scattering densities, but as $N$ is arbitrary, this makes little difference.) For $\mathrm{c}>0$, let $\phi_{\mathrm{c}}$ be supported, say in $\tilde{\mathrm{x}} \leq 2 f(\mathrm{c})$, identically 1 near the region $\tilde{\mathrm{x}} \leq f(\mathrm{c})$; one may assume that $\phi \equiv 1$ on $\operatorname{supp} \phi_{\mathrm{c}}$ by making $\mathrm{c}>0$ small. Then, by Schur's lemma, $\phi_{\mathrm{c}} \phi E_{\mathrm{c}} \phi \phi_{\mathrm{c}}$, acting say on $L_{\mathrm{sc}}^{2}(\overline{\hat{\Omega}})$ (i.e., the $L^{2}$-space relative to scattering densities) is bounded by $C_{N}^{\prime} f(\mathrm{c})^{N}$ for any $N$. Thus, there is $\mathrm{c}_{1}>0$ such that Id $+\phi_{\mathrm{c}} \phi E_{\mathrm{c}} \phi \phi_{\mathrm{c}}=\mathrm{Id}+\phi_{\mathrm{c}} E_{\mathrm{c}} \phi_{\mathrm{c}}$ is invertible for $0<\mathrm{c}<\mathrm{c}_{1}$ on $L_{\mathrm{sc}}^{2}(\overline{\hat{\Omega}})$. (Notice here that this requires the smallness of a seminorm of $\phi_{\mathrm{c}} \phi E_{\mathrm{c}} \phi \phi_{\mathrm{c}}$ in the Fréchet topology of pseudo-differential operators; the continuity discussed above means that that this requires the $C^{k}$-closeness of $\Gamma_{ \pm}$to $\Gamma_{ \pm}^{0}$ for some $k$.) In particular, for $v$ supported in $\tilde{\mathrm{x}} \leq f(\mathrm{c})$, so $\phi_{\mathrm{c}} v=v,\left(\operatorname{Id}+\phi_{\mathrm{c}} E_{\mathrm{c}} \phi_{\mathrm{c}}\right) v=\phi_{\mathrm{c}} G_{\mathrm{c}} T_{\mathrm{c}} v$, so inverting the factor on the left and then undoing the transformation $\Phi_{\mathrm{c}}$ gives the desired conclusion (3.7).

Thus, with $u=\left(u_{0}, \tilde{u}\right)$, we have, with all norms being $L_{\mathrm{sc}}^{2}(\overline{\hat{\Omega}})$-norms,

$$
\begin{aligned}
\left\|\Lambda P_{\digamma} u\right\|^{2} & =\left\|\Lambda P_{0} u_{0}+\Lambda \tilde{P} \tilde{u}\right\|^{2} \\
& =\|\Lambda \tilde{P} \tilde{u}\|^{2}+\left\langle\Lambda \tilde{P} \tilde{u}, \Lambda P_{0} u_{0}\right\rangle+\left\langle\Lambda P_{0} u_{0}, \Lambda \tilde{P} \tilde{u}\right\rangle+\left\langle\Lambda P_{0} u_{0}, \Lambda P_{0} u_{0}\right\rangle .
\end{aligned}
$$

By (3.7), $\|\Lambda \tilde{P} \tilde{u}\| \geq C_{0}\|\tilde{u}\|, C_{0}>0$. On the other hand, $\left\|\Lambda P_{0} u_{0}\right\| \leq C_{1}\left\|u_{0}\right\|$, $\|\Lambda \tilde{P} \tilde{u}\| \leq C_{1}\|\tilde{u}\|$ as elements of $\Psi_{\mathrm{sc}}^{0,0}(\Omega)$ are $L^{2}$-bounded. (By the continuity of the map from $\Gamma_{ \pm}$to $\Lambda P_{0}$ in the appropriate Fréchet topologies, again there is $k$ such that $C_{1}$ is uniform when $\Gamma_{ \pm}$is $C^{k}$-close to $\Gamma_{ \pm}^{0}$.) Using the Cauchy-Schwartz inequality, for $\delta>0$,

$$
\left|\left\langle\Lambda P_{0} u_{0}, \Lambda \tilde{P} \tilde{u}\right\rangle\right| \leq \frac{\delta}{2}\|\tilde{u}\|^{2}+\frac{C_{1}^{2}}{2 \delta}\left\|u_{0}\right\|^{2} .
$$

Thus, the last three terms are bounded by $\delta\|\tilde{u}\|^{2}+\left(1+\delta^{-1}\right) C_{1}^{2}\left\|u_{0}\right\|^{2}$ in absolute value, so we conclude that, with $\delta=C_{0}^{2} / 2$,

$$
\frac{C_{0}^{2}}{2}\|\tilde{u}\|^{2} \leq\left\|\Lambda P_{\digamma} u\right\|^{2}+C_{1}^{2}\left(1+2 C_{0}^{-2}\right)\left\|u_{0}\right\|^{2},
$$

completing the proof of the corollary if $s=r=0$.

\footnotetext{
${ }^{6}$ If we are interested in the region $\rho \geq \rho_{0}, \rho_{0}<0$, within $\overline{\hat{\Omega}}$, with $\rho_{0}$ to be specified, we take, e.g., $\rho_{0}=-c$ in the discussion below, and the desired continuous $f$ with $f(0)=0$, and in this case with $\mathrm{x}_{\mathrm{c}} \leq f(\mathrm{c})$ on $\hat{\Omega}_{\mathrm{c}} \cap\left\{\rho \geq \rho_{0}\right\}$, still exists.
} 
The general case follows via conjugating $P_{\digamma}$ by an elliptic, invertible, element of $\Psi_{\mathrm{sc}}^{s, r}(\overline{\hat{\Omega}})$, which is thus an isomorphism from $H_{\mathrm{sc}}^{s, r}(\overline{\hat{\Omega}})$ to $L^{2}=H_{\mathrm{sc}}^{0,0}(\overline{\hat{\Omega}})$. Note that such a conjugation does not change the principal symbol, and thus the ellipticity.

We now remark that the even simpler setting of the scalar transform with a positive weight $A$ on $S^{*} M, I_{A}$, which was not considered in [49, and which can be considered a special case of $\tilde{J}$ with $\left(u_{0}, u_{1}, \ldots, u_{n}\right)$, is simply replaced by $u$. Thus, for consistency with the above notation, let $\tilde{A}(\mathrm{x}, \mathrm{y}, \lambda, \omega)$ be the weight on $S M$ induced by the metric identification, and let

$$
\left(I_{A} f\right)(\beta)=\int_{\mathbb{R}} \tilde{A}\left(\gamma_{\beta}(t), \gamma_{\beta}^{\prime}(t)\right) f\left(\gamma_{\beta}(t)\right) \mathrm{d} t, \beta \in S M
$$

Then the above argument gives that $P_{\digamma} \in \Psi_{\mathrm{sc}}^{-1,0}(\overline{\hat{\Omega}})$. If at $S_{\partial M} M$, the weight $A$ is independent of the momentum variable $\xi$, it further gives that $P_{\digamma}$ is elliptic in a neighborhood of $\bar{\Omega}$. More generally, a modification of the argument of [49] due to Zhou 52 allows one to show that the principal symbol is fully elliptic in $\bar{\Omega}$ in the scattering sense merely assuming that $A$ is positive (but not the independence condition just mentioned). To see this, one has to Fourier transform (3.5) in $(X, Y)$ with $\tilde{A}_{i}^{j}$ replaced by $\tilde{A}$. The $X$-Fourier transform is unaffected by the presence of $\tilde{A}$ and gives, as in [49, Equation (3.16)],

$$
|Y|^{2-n} e^{-\alpha(\digamma+i \xi)|Y|^{2}} \hat{\chi}(\xi-i \digamma) \tilde{A}(0, \mathrm{y}, 0, \hat{Y}) .
$$

Replacing $\chi$ with a Gaussian, $\chi(s)=e^{-s^{2} /(2 \nu)}, \nu=\digamma^{-1} \alpha$, which does not have compact support, but an approximation argument (in symbols of order -1 ) will give this desired property, and one can compute that this is, up to a constant factor,

$$
\digamma^{-1 / 2} \alpha^{1 / 2}|Y|^{2-n} e^{-\digamma^{-1}\left(\xi^{2}+\digamma^{2}\right) \alpha|Y|^{2} / 2} \tilde{A}(0, \mathrm{y}, 0, \hat{Y}) .
$$

We need to compute the Fourier transform in $Y$. Following [52], one expresses this in polar coordinates in $Y$,

$$
\begin{aligned}
& \digamma^{-1 / 2} \int_{\mathbb{S}^{n-2}} \int_{0}^{\infty} e^{-i|Y| \hat{Y} \cdot \eta} \alpha^{1 / 2} e^{-\digamma^{-1}\left(\xi^{2}+\digamma^{2}\right) \alpha|Y|^{2} / 2} \tilde{A}(0, \mathrm{y}, 0, \hat{Y}) \mathrm{d}|Y| \mathrm{d} \hat{Y} \\
& =\frac{1}{2} \digamma^{-1 / 2} \int_{\mathbb{S}^{n-2}} \int_{\mathbb{R}} e^{-i t \hat{Y} \cdot \eta} \alpha^{1 / 2} e^{-\digamma^{-1}\left(\xi^{2}+\digamma^{2}\right) \alpha t^{2} / 2} \tilde{A}(0, \mathrm{y}, 0, \hat{Y}) \mathrm{d} t \mathrm{~d} \hat{Y}
\end{aligned}
$$

which in turn becomes, up to a constant factor,

$$
\left(\xi^{2}+\digamma^{2}\right)^{-1 / 2} \int_{\mathbb{S}^{n-2}} e^{-\digamma|\hat{Y} \cdot \eta|^{2} /\left(2\left(\xi^{2}+\digamma^{2}\right) \alpha\right)} \tilde{A}(0, \mathrm{y}, 0, \hat{Y}) \mathrm{d} \hat{Y} .
$$

The integrand is now positive, which gives the desired ellipticity at $x=0$. (One also needs to check the ellipticity as $(\xi, \eta) \rightarrow \infty$; this is standard, see [49,52.) One proceeds with an approximation argument as in [49,52] to complete the proof of the ellipticity. Thus, the above argument gives the estimate

$$
\|f\|_{H_{\mathrm{sc}}^{s, r}(\overline{\hat{\Omega}})} \leq C\left\|P_{\digamma} f\right\|_{H_{\mathrm{sc}}^{s+1, r}(\overline{\hat{\Omega}})} .
$$

As discussed in [49] after Lemma 3.6, this yields the following corollary. 
Corollary 3.2. The weighted scalar transform with a positive weight $A$ on $S^{*} M$, with $\tilde{A}$ the associated weight on $S M$,

$$
\left(I_{A} f\right)(\beta)=\int_{\mathbb{R}} \tilde{A}\left(\gamma_{\beta}(t), \gamma_{\beta}^{\prime}(t)\right) f\left(\gamma_{\beta}(t)\right) \mathrm{d} t,
$$

satisfies that for $\digamma>0$ there is $\mathrm{c}_{0}>0$ such that for $\delta>0,0<\mathrm{c}<\mathrm{c}_{0}, s \geq-1$, we have

$$
\|f\|_{e^{(\digamma+\delta) / \times} H^{s}\left(\Omega_{c}\right)} \leq C\left\|I_{A} f\right\|_{e^{\digamma / \times} H^{s+1}\left(\mathcal{M}_{c}\right)} .
$$

We now return to the actual case of interest and apply Corollary 3.1 with $u_{0}=$ $e^{-\digamma / x} f, u_{j}=e^{-\digamma / x} \partial_{j} f$. If we show that (uniformly in $\Gamma_{ \pm}$, which are indeed irrelevant for this argument) given $\tilde{\delta}>0$, there is $\mathrm{c}_{0}>0$ such that $\left\|u_{0}\right\|_{L^{2}} \leq \tilde{\delta}\|\tilde{u}\|_{L^{2}}$; i.e., $e^{-\digamma / \times} f$ is bounded by a small multiple of a derivative of $f$ times $e^{-\digamma / x}$ in $L^{2}$, when $f$ is supported in $\overline{\Omega_{\mathrm{c}}}, 0<\mathrm{c}<\mathrm{c}_{0}$, and then for $\tilde{\delta}>0$ sufficiently small (3.6) proves that if $P_{\digamma} u$ vanishes, then so does $u$. That is, in this case so does $f$, for the $u_{0}$ term can then be absorbed into the left-hand side of (3.6),

$$
(1-\tilde{\delta} C)\|u\|_{H_{\mathrm{sc}}^{0,0}(\overline{\hat{\Omega}})} \leq C\left\|P_{\digamma} u\right\|_{H_{\mathrm{sc}}^{1,0}(\overline{\hat{\Omega}})} .
$$

Further, rewriting this by removing the weights $e^{-\digamma / x}$, and estimating the norms in terms of the standard $L^{2}$-based space (cf. the discussion after [49, Lemma 3.6] already referenced above) 7 gives, for $\delta>0$,

$$
\|f\|_{e^{(\digamma+\delta) / \times} H^{1}\left(\Omega_{c}\right)} \leq C\left\|\tilde{J}\left(f, \partial_{1} f, \ldots, \partial_{n} f\right)\right\|_{e^{\digamma / \times} H^{1}\left(\mathcal{M}_{c}\right)},
$$

with $C$ uniform for $\Gamma_{ \pm} C^{k}$-close to $\Gamma_{ \pm}^{0}$.

But this can now easily be done: let $V$ be a smooth vector field with $V \times=0$, so $V$ is tangent to the boundary of $\Omega_{\mathrm{c}}$ for every c, and make the non-degeneracy assumption that, for some $c_{1}>0$, there is a continuous $T:\left[0, c_{1}\right) \rightarrow \mathbb{R}$ such that $T(0)=0$ and the $V$-flow takes every point in $\overline{\Omega_{\mathrm{c}}}$ to $\partial M$ in time $\leq T$ (c) (i.e., outside the original manifold). Then the Poincaré inequality for $V$ gives 8

$$
\|v\|_{L_{\mathrm{sc}}^{2}} \leq C_{2} T(\mathrm{c})\|V v\|_{L_{\mathrm{sc}}^{2}},
$$

for $v$ vanishing outside $M$; hence the constant is small if $T(\mathrm{c})>0$ is small. (Here the $L^{2}$ space we need is the scattering $L^{2}$-space, $L_{\mathrm{sc}}^{2}$, which is $\mathrm{x}^{(n+1) / 2}$ times the standard $L^{2}$-space, but the extra weight does not affect the argument, since $V$ commutes with multiplication by powers of $\mathrm{x}$.)

To see (3.11), we recall a standard proof of the local Poincaré inequality: in order to reduce confusion with the notation, let $\left(z_{1}, \ldots, z_{n}\right)=\left(z^{\prime}, z_{n}\right)$ be the coordinates, $z_{1}=0$ being the boundary of $\Omega$ (so $\times$ would be $z_{1}$ ), and assume that the flow of $\partial_{z_{n}}$ flows from every point in $\Omega$ to outside the region in "time" $\leq \delta$. To normalize the argument, assume that $z_{n} \geq 0$ in $\Omega$, and we want to estimate $v$ in $z_{n} \leq \delta$. We assume that the $L^{2}$ space is given by a density $F\left(z^{\prime}\right)|d z|$. Then, for $v \in C^{\infty}\left(\mathbb{R}_{+}^{n}\right)$, $\mathbb{R}_{+}^{n}=[0, \infty)_{z_{1}} \times \mathbb{R}^{n-1}$, with support in $z_{n} \geq 0$, by the fundamental theorem of

\footnotetext{
${ }^{7}$ The $\delta$ loss is actually just the loss of a power of $\mathrm{x}$, due to the change of the measure.

${ }^{8}$ Notice that our treatment of the $\tilde{x}$-dependence of the problem relies on reducing to a model, where $\tilde{x}$ is replaced by a fixed function $\underline{\tilde{x}}$, so the following argument is, in fact, uniform in $\tilde{x}$.
} 
calculus and the Cauchy-Schwartz inequality,

$$
\begin{aligned}
\left|v\left(z^{\prime}, z_{n}\right)\right|=\left|\int_{0}^{z_{n}} \partial_{n} v\left(z^{\prime}, t\right) \mathrm{d} t\right| & \leq\left(\int_{0}^{z_{n}} 1 \mathrm{~d} t\right)^{1 / 2}\left(\int_{0}^{z_{n}}\left|\partial_{n} v\left(z^{\prime}, t\right)\right|^{2} \mathrm{~d} t\right)^{1 / 2} \\
& \leq \delta^{1 / 2}\left(\int_{0}^{\delta}\left|\partial_{n} v\left(z^{\prime}, t\right)\right|^{2} \mathrm{~d} t\right)^{1 / 2} .
\end{aligned}
$$

Squaring both sides, multiplying by $F\left(z^{\prime}\right)$, and integrating in $z^{\prime}, z_{n}$ (to $z_{n}=\delta$ ) gives

$$
\int_{z_{n} \leq \delta}\left|v\left(z^{\prime}, z_{n}\right)\right|^{2} F\left(z^{\prime}\right) \mathrm{d} z \leq \delta^{2} \int_{t \leq \delta}\left|\partial_{n} v\left(z^{\prime}, t\right)\right|^{2} \mathrm{~d} t F\left(z^{\prime}\right) \mathrm{d} z^{\prime} .
$$

This says that actually

$$
\|v\|_{L^{2}\left(\mathbb{R}_{+}^{n} ; F\left(z^{\prime}\right)|\mathrm{d} z|\right)} \leq \delta\left\|\partial_{n} v\right\|_{L^{2}\left(\mathbb{R}_{+}^{n} ; F\left(z^{\prime}\right)|\mathrm{d} z|\right)},
$$

proving the claim (using $F\left(z^{\prime}\right)=z_{1}^{-n-1}$ ) in view of the quasi-isometry invariance (which gives a constant factor) of the bound (3.11). Even if there is more complicated topology, so there are no global coordinates and vector fields as stated, dividing up the problem into local pieces and adding them together gives the desired result: taking steps of size $\delta$, one needs $T / \delta$ steps to cover the set, using cutoff functions to localize is easily seen to give a bound proportional to $T$.

On the other hand, in view of the strict convexity of the boundary, one can construct such a $V$ and $T$. With $v=e^{-\digamma / \times} f$, this is exactly the desired conclusion since $V\left(e^{-\digamma / \times} f\right)=e^{-\digamma / \times} V f$, namely (3.9), and thus (3.10), hold.

In fact, we can prove the analogue of (3.9) on stronger spaces,

$$
\|u\|_{H_{\mathrm{sc}}^{s, 0}(\overline{\hat{\Omega}})} \leq C\left\|P_{\digamma} u\right\|_{H_{\mathrm{sc}}^{s+1,0}(\overline{\hat{\Omega}})}, \quad s \geq 0,
$$

which in turn gives, for $s \geq 0$,

$$
\|f\|_{e^{(\digamma+\delta) / x} H^{s+1}\left(\Omega_{c}\right)} \leq C\left\|\tilde{J}\left(f, \partial_{1} f, \ldots, \partial_{n} f\right)\right\|_{e^{\digamma / \times} H^{s+1}\left(\mathcal{M}_{c}\right)},
$$

with $C$ uniform for $\Gamma_{ \pm} C^{k}$-close to $\Gamma_{ \pm}^{0}$. To see this, notice that (3.6) is an elliptic estimate when we have $u_{0}=e^{-\digamma / \times} f, u_{j}=e^{-\digamma / \times} \partial_{j} f$, for

$$
\|u\|_{H_{\mathrm{sc}}^{s, r}(\overline{\hat{\Omega}})} \leq C\left\|P_{\digamma} u\right\|_{H_{\mathrm{sc}}^{s+1, r}(\overline{\hat{\Omega}})}+C\left\|u_{0}\right\|_{H_{\mathrm{sc}}^{s, r}(\overline{\hat{\Omega}})}
$$

implies that 9

$$
\left\|e^{-\digamma / \times} f\right\|_{H_{\mathrm{sc}}^{s+1, r}(\overline{\hat{\Omega}})} \leq C\left\|P_{\digamma} e^{-\digamma / \times}(f, d f)\right\|_{H_{\mathrm{sc}}^{s+1, r}(\overline{\hat{\Omega}})}+C\left\|e^{-\digamma / \times} f\right\|_{H_{\mathrm{sc}}^{s, r}(\overline{\hat{\Omega}})} .
$$

For $s=1, r=0$, we have the second term on the right-hand side controlled by $P_{\digamma} e^{-\digamma / \times}(f, d f)$ in $H_{\mathrm{sc}}^{1,0}$ in view of (3.9), so the $H_{\mathrm{sc}}^{2,0}$-norm of $e^{-\digamma / \times} f$ is, in fact, controlled by $\left\|P_{\digamma} e^{-\digamma / \times}(f, d f)\right\|_{H_{\mathrm{sc}}^{2,0}(\overline{\hat{\Omega}})}$, which we can iterate further 10 to prove (3.12).

In summary we have proved that with $L^{2}(\Omega)=L^{2}\left(\Omega_{\mathrm{c}}\right)$ the standard $L^{2}$-space now follows (as the exponential weight $e^{-\digamma / x}$ maps such $f$ to $L_{\mathrm{sc}}^{2}(\overline{\hat{\Omega}})$; see also the discussion after Lemma 3.6 in [49]).

\footnotetext{
${ }^{9}$ Notice that the scattering derivatives are actually weaker than the derivatives $\partial_{j}$ entering $u$, and one can absorb the term given by commuting a scattering derivative through $e^{-\digamma / x}$ into the last term on the right-hand side.

${ }^{10}$ We can easily allow $s \geq 0$ non-integer by slightly modifying the argument here.
} 
Proposition 3.3. There is $\mathrm{c}_{0}>0$ such that for $0<\mathrm{c}<\mathrm{c}_{0}$, if $f \in H^{1}\left(\Omega_{\mathrm{c}}\right)$ and $\tilde{J}\left(f, \partial_{1} f, \ldots, \partial_{n} f\right)=0$, then $f=0$.

In fact, for $\digamma>0, s \geq 1$, there exist $\mathrm{c}_{0}>0, k$ and $\epsilon>0$ such that the following holds. For $\delta>0$ there is $C>0$ such that if $0<\mathrm{c}<\mathrm{c}_{0}, \Gamma_{ \pm}$is $\epsilon$-close to $\Gamma_{ \pm}^{0}$ in $C^{k}$, $\tilde{\mathrm{x}}$ is $\epsilon$-close to $\tilde{\mathrm{x}}_{0}$ in $C^{k}$, then

$$
\|f\|_{e^{(\digamma+\delta) / \times} H^{s}\left(\Omega_{\mathrm{c}}\right)} \leq C\left\|\tilde{J}\left(f, \partial_{1} f, \ldots, \partial_{n} f\right)\right\|_{e^{\digamma / \times} H^{s}\left(\mathcal{M}_{\mathrm{c}}\right)} .
$$

Moreover, with $\Omega_{\mathrm{c}, \rho_{0}}=\hat{\Omega}_{\mathrm{c}} \cap\left\{\rho \geq \rho_{0}\right\}$, and $\mathcal{M}_{\mathrm{c}, \rho_{0}}$ being defined analogously to $\mathcal{M}_{\mathrm{c}}$ with $\partial M=\{\rho=0\}$ being replaced by $\left\{\rho=\rho_{0}\right\}$, we have the following: for $\digamma>0$ and $s \geq 1$ there exist $\mathrm{c}_{0}>0, \rho_{0}<0, k$, and $\epsilon>0$ such that the following holds. For $\delta>0$ there is $C>0$ such that if $0<\mathrm{c}<\mathrm{c}_{0}, \Gamma_{ \pm}$is $\epsilon$-close to $\Gamma_{ \pm}^{0}$ in $C^{k}$, $\tilde{\mathrm{x}}$ is $\epsilon$-close to $\tilde{\mathrm{x}}_{0}$ in $C^{k}$, then $f \in H^{s+1}\left(\Omega_{\mathrm{c}, \rho_{0}}\right)$ implies that

$$
\left.\|f\|_{e^{(\digamma+\delta) / \times} H^{s}\left(\Omega_{c, \rho_{0}}\right)} \leq C\left\|\tilde{J}\left(f, \partial_{1} f, \ldots, \partial_{n} f\right)\right\|_{e^{\digamma} / \times H^{s}\left(\mathcal{M}_{c}, \rho_{0}\right.}\right) .
$$

\section{Proofs of Theorems 1.1 1.4}

Proof of Theorem 1.2. Let $c$ and $\tilde{c}$ be as in the theorem. Redefine the scattering relation $\mathcal{L}$ as in Figure 1. By Proposition 2.1, we get $J_{j} f(\gamma)=0$; see (2.12) for all geodesics close enough to the ones tangent to $\partial M$ at $p$. The weights are given by (2.14), in the new parameterization, with the ellipticity condition satisfied by (2.15). Then Proposition 3.3 implies $f=0$ in a neighborhood of $p$, where $f=c^{2}-\tilde{c}^{2}$ as in (2.8).

Proof of Theorem 1.1. Note first that we can complete $\left(M, c_{0}^{-2}\right)$, and similarly $\left(M, \tilde{c}^{-2} g_{0}\right)$ to compact Riemannian manifolds without boundary. Then we can choose a neighborhood of $p$ small enough so that the exponential map based on any point of that neighborhood is a local diffeomorphism for short enough vectors, both for $c$ and for $\tilde{c}$. This implies that there is a neighborhood $U$ of $p$ so that the distance $d\left(p_{1}, p_{2}\right)$ between any two points $p_{1}, p_{2}$ in $U$ is realized by $\left|\exp _{p_{1}}^{-1}\left(p_{2}\right)\right|$, related to the first and the second metrics, respectively, where exp is the localized exponential map as above. We can easily recover $c$ and $\tilde{c}$ on $\partial M \cap U$ by taking the limit $p_{1} \rightarrow p_{2}$. As Michel proved [21, for simple manifolds, the scattering elation can be recovered by differentiating the distance function; see also [38, Section 2]. This applies to our case as well because $U$ is small enough. Then the proof follows from Theorem 1.2 .

Proof of Theorem 1.4. Theorem 1.4 is now an easy consequence of Theorem 1.1 using a layer stripping argument. Let $f=c^{2}-\tilde{c}^{2}$. Assume $f \neq 0$, then supp $f$ has a non-empty interior. On the other hand, let $\tau=\inf _{\text {supp } f} \rho$; if $\tau=T$, we are done, for then supp $f \subset M \backslash \cup_{t \in[0, T)} \Sigma_{t}$. Thus, suppose $\tau<T$, so $f \equiv 0$ on $\Sigma_{t}$ for $t<\tau$, but there exists $x \in \Sigma_{\tau} \cap \operatorname{supp} f$ (since supp $f$ is closed). We will show below how to use Theorem 1.2 on $M_{\tau}:=\rho^{-1}(\tau, \infty)$ to conclude that a neighborhood of $x$ is disjoint from supp $f$ to obtain a contradiction.

All we need to show is that the scattering relations $L_{\tau}$ and $\tilde{L}_{\tau}$ on $\Sigma_{\tau}$ coincide. Note that $\Sigma_{\tau}=\partial M_{\tau}$ is strictly convex for $\tilde{g}$ as well because the second fundamental form for $\tilde{g}$ can be computed by taking derivatives from the exterior $\rho<\tau$, where $g=\tilde{g}$. Fix $\left(x_{\tau}, v_{\tau}\right) \in \partial_{-} S M_{\tau}$; see Figure 2. The geodesic $\gamma_{x_{\tau}, v_{\tau}}(s)$ cannot hit $\Sigma_{\tau}$ again for negative "times" $s$ because, otherwise, we would get a contradiction with the strict convexity at $\Sigma_{t}$, where $t$ corresponds to the smallest value of $\rho$ on 
that geodesic between two contacts with $\Sigma_{\tau}$. Since $c=\tilde{c}$ outside $M_{\tau}, \gamma_{x_{\tau}, v_{\tau}}(s)$ and $\tilde{\gamma}_{x_{\tau}, v_{\tau}}(s)$ coincide outside $M_{\tau}$ for $s<0$. Proposition 5.1 below shows that this negative geodesic ray must be non-trapping; i.e., $\gamma_{x_{\tau}, v_{\tau}}$ would hit $\partial M$ for a finite negative time $s$ at some point and direction $(x, v) \in \partial_{-} S M$. In the same way, we show that the same holds for the positive part, $s>0$, of a geodesic issued from $L_{\tau}\left(x_{\tau}, y_{\tau}\right)=:\left(y_{\tau}, w_{\tau}\right) \in \partial_{+} S M_{\tau}$; and the corresponding point on $\partial_{+} S M$ will be denoted by $(y, w)$. Then, since $L(x, v)=(y, w)$, we would also get $L_{\tau}\left(x_{\tau}, v_{\tau}\right)=\left(y_{\tau}, w_{\tau}\right)=\tilde{L}_{\tau}\left(x_{\tau}, v_{\tau}\right)$.

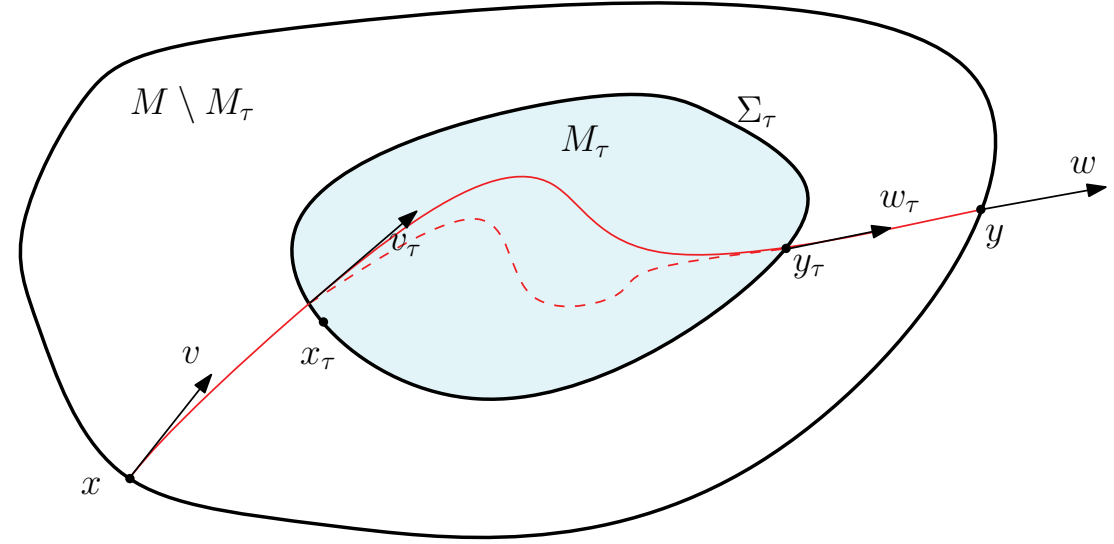

FiguRE 2. One can recover the scattering relation on $\Sigma_{\tau}$ knowing that on $\partial M$.

\section{Stability ANALYSis}

In this section, we prove the stability estimate in Theorem 1.5 and a global stability estimate, see Theorem 5.2 below. We follow some of the ideas in [41, section 7].

5.1. Boundary stability. We start with stability at the boundary.

Theorem 5.1. Let $c$ and $\tilde{c}$ be such that $\partial M$ is strictly convex w.r.t. both $c^{-2} g_{0}$ and $\tilde{c}^{-2} g_{0}$, and let $\Gamma \subset \subset \Gamma^{\prime} \subset \partial M$ be two sufficiently small open subsets of the boundary. Then

$$
\left\|\partial_{x^{n}}^{k}(c-\tilde{c})\right\|_{C^{m}(\bar{\Gamma})} \leq C_{k, m}\left\|d^{2}-\tilde{d}^{2}\right\|_{C^{m+2 k+2}\left(\overline{\Gamma^{\prime} \times \Gamma^{\prime}}\right)},
$$

where $C_{k, m}$ depends only on $\Omega$ and on an upper bound of $c, c^{-1}, \tilde{c}, \tilde{c}^{-1}$ in $C^{m+2 k+5}(M)$.

Proof. We know from [41, Section 7] that it is true for the metrics in boundary normal coordinates. More precisely, let $\left(x^{\prime}, x^{n}\right)$ be boundary local coordinates for $g$, i.e., locally,

$$
c^{-2} g_{0}=h_{\alpha \beta} \mathrm{d} x^{\alpha} \mathrm{d} x^{\beta}+\left(\mathrm{d} x^{n}\right)^{2}=: h_{i j} \mathrm{~d} x^{i} \mathrm{~d} x^{j} .
$$

Note that the notational convention is different than the one in Section 3. $(\mathrm{x}, \mathrm{y})$ is now replaced by $\left(x^{\prime}, x^{n}\right)$, and $x$ and $y$ are generic points in (the local chart) in $\mathbb{R}^{n}$. We use the convention that Greek indices run from 1 to $n-1$, while Latin ones 
run from 1 to $n$. Let $\psi$ be the diffeomorpishm fixing the boundary pointwise near $p$, i.e., $\psi\left(x^{\prime}, 0\right)=\left(x^{\prime}, 0\right)$, so that

$$
\psi^{*}\left(\tilde{c}^{-2} g_{0}\right)=\tilde{h}_{\alpha \beta} \mathrm{d} x^{\alpha} \mathrm{d} x^{\beta}+\left(\mathrm{d} x^{n}\right)^{2}
$$

near $p$. Then (5.1) holds for $h-\tilde{h}$.

We have, with $y=\psi(x)$,

$$
\psi^{*}\left(\tilde{c}^{-2} g_{0}\right)(x)=\left(c^{2} \tilde{c}^{-2}\right)(y(x)) h_{i^{\prime} j^{\prime}}(y(x)) \frac{\partial y^{i^{\prime}}}{\partial x^{i}} \frac{\partial y^{j^{\prime}}}{\partial x^{j}} \mathrm{~d} x^{i} \mathrm{~d} x^{j} .
$$

In particular, by (5.2) and (5.3),

$$
\left(c^{2} \tilde{c}^{-2}\right)(y(x)) h_{i^{\prime} j^{\prime}}(y(x)) \frac{\partial y^{i^{\prime}}}{\partial x^{\alpha}} \frac{\partial y^{j^{\prime}}}{\partial x^{\beta}}=\tilde{h}_{\alpha \beta}(x),
$$

which can be written as

$$
\left(\left(c^{2} \tilde{c}^{-2}\right)(y(x))-1\right) h_{i^{\prime} j^{\prime}}(y(x)) \frac{\partial y^{i^{\prime}}}{\partial x^{\alpha}} \frac{\partial y^{j^{\prime}}}{\partial x^{\beta}}=\tilde{h}_{\alpha \beta}(x)-h_{i^{\prime} j^{\prime}}(y(x)) \frac{\partial y^{i^{\prime}}}{\partial x^{\alpha}} \frac{\partial y^{j^{\prime}}}{\partial x^{\beta}} .
$$

We have

$$
\partial y^{i} / \partial x^{\beta}=\delta_{\beta}^{i} \text { for } x^{n}=0,
$$

which, of course, can be further differentiated w.r.t. tangential variables $x^{\alpha}$. Therefore,

$$
\left.\left(\tilde{c}^{-2} c^{2}-1\right) h_{\alpha \beta}\right|_{x^{n}=0}=\left.\left(\tilde{h}_{\alpha \beta}-h_{\alpha \beta}\right)\right|_{x^{n}=0} .
$$

Since $\left\{h_{\alpha \beta}\right\}$ is invertible (at least for one multi-index is enough), we get (5.1) for $\tilde{c}^{-2} c^{2}-1$, and therefore for $\tilde{c}-c$ for $k=0$.

In order to do the same for $k=1$, we need to estimate $\partial(y-x) / \partial x^{n}$ at $x^{n}=0$ first. The diffeomorphism $\psi$ identifies boundary normal coordinates for $g$ and $\tilde{g}$. The normal $\nu=(0,1)$ (here, 0 is $n-1$ dimensional) is the unit for $g=c^{-2} g_{0}$, but it has length $\tilde{c}^{-1} c$ in the metric $\tilde{g}$. The inner unit vector in the metric $\tilde{g}$ is therefore $\tilde{\nu}=\tilde{c} c^{-1}(0,1)$; hence

$$
y=\tilde{\gamma}_{\left(x^{\prime}, 0\right),\left(0, \tilde{c} c^{-1}\right)}\left(x^{n}\right) .
$$

Differentiate this at $x^{n}=0$ to get that it depends only on $\tilde{c} c^{-1}$ on $x^{n}=0$ but not on its derivatives; in fact,

$$
\left.\frac{\partial y}{\partial x^{n}}\right|_{x^{n}=0}=\left(0, \tilde{c} c^{-1}\right)
$$

and this can be differentiated w.r.t. $x^{\prime}$. Note that we can get the same result by comparing the $\alpha n$ metric elements in (5.2) and (5.3). Then $\partial(y-x) /\left.\partial x^{n}\right|_{x^{n}=0}=$ $\left(0, \tilde{c} c^{-1}-1\right)$. By what we proved, the latter satisfies (5.1).

Differentiate (5.5) with respect to $x^{n}$ at $x^{n}=0$. Using (5.8), we get

$$
\begin{gathered}
\left.\tilde{c} c^{-1} h_{\alpha \beta} \frac{\partial\left(c^{2} \tilde{c}^{-2}-1\right)}{\partial x^{n}}\right|_{x^{n}=0} \\
=-\left.\left(c^{2} \tilde{c}^{-2}\left(x^{\prime}, 0\right)-1\right) \frac{\partial}{\partial x^{n}}\right|_{x^{n}=0}\left(h_{i^{\prime} j^{\prime}}(y(x)) \frac{\partial y^{i^{\prime}}}{\partial x^{\alpha}} \frac{\partial y^{j^{\prime}}}{\partial x^{\beta}}\right) \\
+\left.\frac{\partial}{\partial x^{n}}\right|_{x^{n}=0}\left(\tilde{h}_{\alpha \beta}(x)-h_{i^{\prime} j^{\prime}}(y(x)) \frac{\partial y^{i^{\prime}}}{\partial x^{\alpha}} \frac{\partial y^{j^{\prime}}}{\partial x^{\beta}}\right) .
\end{gathered}
$$


To estimate the last derivative, notice that by (5.8),

$$
\left.\frac{\partial^{2} y}{\partial x^{n} \partial x^{\alpha}}\right|_{x^{n}=0}=\left.\frac{\partial}{\partial x^{\alpha}}\right|_{x^{n}=0}\left(0, \tilde{c} c^{-1}\right),
$$

and we proved the estimate for the tangential derivatives of $\tilde{c} c^{-1}$ (same as the tangential derivatives of $\tilde{c} c^{-1}-1$ ) already. Therefore, the second summand on the r.h.s. of (5.9) can be written as a sum of a term involving the normal derivative of $h-\tilde{h}$, which estimate is known, plus terms involving the first tangential derivatives of $\tilde{c} c^{-1}-1$, which we estimated already. We can now deduce the desired estimate for $\partial\left(c^{2} \tilde{c}^{-2}-1\right) / \partial x^{n}$ at $x^{n}=0$. Differentiate again (5.5) and (5.7) with respect to $x^{n}$ at $x^{n}=0$, to prove the estimate for $k=2$, etc.

\subsection{Local interior stability, proof of Theorem 1.5. Set}

$$
\delta:=\left\|d^{2}-\tilde{d}^{2}\right\|_{C}\left(\overline{\Gamma^{\prime} \times \Gamma^{\prime}}\right) .
$$

We use below interpolation estimates in the $C^{k}$ spaces; see, e.g., 48. If $m<k$ (see (1.2)), we have

$$
\|c-\tilde{c}\|_{C^{m}(U)} \leq C \varepsilon_{0}^{\mu},
$$

with some $0<\mu=\mu(m) \leq 1$. Also, by Theorem [5.1, for any $m$,

$$
\left\|d^{2}-\tilde{d}^{2}\right\|_{C^{m}\left(\overline{\Gamma^{\prime} \times \Gamma^{\prime}}\right)} \leq C \delta^{\mu}
$$

with another $0<\mu=\mu(m) \leq 1$, if $k \gg 1$, under the a priori estimate (1.2) of the theorem. We will use the smallest $\mu$ above, and in the proof below, we will not specify the values of $k$ and $\mu$ which are guaranteed to work, even though this can be done. In principle, increasing $k$ in the theorem (assuming a stronger a priori bound) increases $\mu$ (makes the Hölder stability estimate stronger), and vice versa.

We extend $c$ and $\tilde{c}$ outside $M$ by preserving the $\delta^{\mu}$-closeness on $\Gamma$. Extend $c$ in a smooth way first. As above, let $\left(x^{\prime}, x^{n}\right)$ be boundary normal coordinates so that $x^{n}$ is the distance in the metric $g_{0}$ to $\partial M$, positive outside $M$. Given an integer $k$, let $E_{k}(\tilde{c}-c)$ be the truncated Taylor series of $\tilde{c}-c$ w.r.t. $x^{n}$ at $x^{n}=0$ of degree $m$, for $x^{n}>0$.

Let $0<-\rho_{0} \ll 1, \mathrm{c}>0$ be such that the estimate in Proposition 3.3 holds in $\Omega_{\mathrm{c}, \rho_{0}}$, with the choice of a boundary defining function $\rho=-x^{n}$. We can also assume that Proposition 3.3 holds for all sound speeds $c$ as in (1.2), with $A$ fixed, and $\varepsilon_{0} \ll 1$. Set

$$
\tilde{c}_{1}= \begin{cases}\tilde{c} & x^{n}<0 \\ c+\chi\left(x^{n} /\left|\rho_{0}\right|\right) E_{k}(\tilde{c}-c), & x^{n}>0\end{cases}
$$

where $\chi(t)=1$ for $t<1 / 4$, and $\chi(t)=0$ for $t \geq 1 / 2$. Then $\tilde{c}_{1}=\tilde{c}$ in $M$, and $\tilde{c}_{1}(x)=c(x)$ when $d(x, M)>\left|\rho_{0}\right| / 2$. Moreover, for any small enough neighborhood $U$ of $p$,

$$
\left\|c-\tilde{c}_{1}\right\|_{C^{m}(U \backslash M)} \leq C\|c-\tilde{c}\|_{C^{m}(\partial M)} \leq C \delta^{\mu} .
$$

We drop the subscript 1 and denote the $\tilde{c}_{1}$ by $\tilde{c}$ below.

Next, we compare $L$ and $\tilde{L}$ pushed to $\partial S B\left(x_{0}, r\right)$; see the Figure1. Recall that in Section 2.2, we redefined $L$ and $\tilde{L}$ to act from $U_{-}$to $U_{+}$, where $U_{ \pm} \subset \partial_{ \pm} S B\left(x_{0}, r\right)$. 
It is easy to see that for $x, y$ near $x_{0}, d(x, y) / C \leq \tilde{d}(x, y) \leq C d(x, y)$ with $C$ depending on $A$ in (1.2) only. Then writing $d-\tilde{d}=\left(\bar{d}^{2}-\tilde{d}^{2}\right) /(\bar{d}+\tilde{d})$, we get from (5.12),

$$
\left|\partial_{x^{\prime}, y^{\prime}}^{\alpha}(d(x, y)-\tilde{d}(x, y))\right| \leq C \delta^{\mu} / d(x, y)^{|\alpha|+1} \quad \text { on } \Gamma^{\prime} \times \Gamma^{\prime} .
$$

We are only going to need this for $|\alpha| \leq 1$. Set

$$
\xi(x, y)=-\mathrm{d}_{x} d(x, y), \quad \eta(x, y)=\mathrm{d}_{y} d(x, y),
$$

which are just the tangent unit codirections at $x$ and $y$ of the geodesic connecting those two points. We define $\tilde{\xi}(x, y)$ and $\tilde{\eta}(x, y)$ in a similar way. By the strict convexity,

Since $\xi$ and $\tilde{\xi}$ are units,

$$
\left|\xi_{n}\right| \geq d(x, y) / C
$$

$$
\xi_{n}(x, y)=-\sqrt{c^{-2}(x)-g_{0}^{\alpha \beta}(x) \xi_{\alpha} \xi_{\beta}}, \quad \tilde{\xi}_{n}(x, y)=-\sqrt{\tilde{c}^{-2}(x)-g_{0}^{\alpha \beta}(x) \tilde{\xi}_{\alpha} \tilde{\xi}_{\beta}} .
$$

Then, using (5.14) with $|\alpha|=1$, we get

$$
\left|\xi_{n}(x, y)-\tilde{\xi}_{n}(x, y)\right| \leq C \delta^{\mu} / d(x, y)^{2} .
$$

This, combined with (5.14) for $\alpha=0$, yields

$$
|\xi(x, y)-\tilde{\xi}(x, y)| \leq C \delta^{\mu} / d(x, y)^{2} .
$$

In the same way, we get

$$
\|\eta(x, y)-\tilde{\eta}(x, y)\|_{C^{k-1}} \leq C \delta^{\mu} / d(x, y)^{2} .
$$

For every $(x, y) \in \partial M \times \partial M$, both close to $x_{0}$, consider the shortest geodesics $\gamma_{[x, y]}$ and $\tilde{\gamma}_{[x, y]}$ connecting them, in the metrics $g$ and $\tilde{g}$, respectively. By the a priori estimate (1.2), those two geodesics lie in a neighborhood of $x_{0}$ of size which shrinks to zero, as $d\left(x, x_{0}\right)$ and $d\left(y, x_{0}\right)$ tend to zero, uniformly for all $c$ and $\tilde{c}$ satisfying (1.2). Next, we extend each one of them, lifted to the cotangent bundle (i.e., to the bicharacteristics, called "rays" below), in both directions until they hit $\partial S B\left(x_{0}, r\right)$. We denote by $z_{-}=\left(x_{-}, \xi_{-}\right)$(respectively, $\left.\tilde{z}_{-}=\left(\tilde{x}_{-}, \tilde{\xi}_{-}\right)\right)$the common point with $\partial_{-} S B\left(x_{0}, r\right)$, and by $z_{+}=\left(y_{+}, \eta_{+}\right)$(respectively, $\left.\tilde{z}_{+}=\left(\tilde{y}_{+}, \tilde{\eta}_{+}\right)\right)$the common point with $\partial_{+} S B\left(x_{0}, r\right)$; see Figure 3 .

Let $\Sigma_{-}$be the submanifold of $\partial_{-} S B\left(x_{0}, r\right)$ consisting of all $z$ there such that the bicharacteristic $Z(t, z)$ through it, in the metric $g$, is tangent to $\partial M$ at some point close enough to $x_{0}$. Let $U_{0}$ be the connected neighborhood of it consisting of those points staying at distance (with respect to fixed coordinates) at most $O\left(\delta^{\mu^{\prime}}\right)$ with $0<\mu^{\prime}<\mu$ which we chose below. Then the small but $\delta$-independent neighborhood $U_{-}$of $\Sigma_{-}$admits the decomposition $U_{-}=U_{0}+U_{\text {in }}+U_{\text {out }}$, where $U_{\text {in }}$ and $U_{\text {out }}$ are disconnected components, such that the rays from $U_{\text {in }}$ in the metric $g$ hit $\partial M$, and the rays from $U_{\text {out }}$ do not. All of them are extended until they hit $\partial_{+} S B\left(x_{0}, r\right)$. We denote the corresponding travel times by $\ell(z)$, as in Section 2.2 , but now $\partial M$ is replaced by the geodesic sphere $\partial B\left(x_{0}, r\right)$.

The rays issued from $U_{\text {out }}$ would hit $\partial_{+} S B\left(x_{0}, r\right)$ and miss $\partial M$ by definition. They will stay at a distance at least $\delta^{\mu^{\prime}} / C$ from $\partial M$. The same is true for the rays issued from $U_{\text {out }}$ related to the metric $\tilde{g}=\tilde{c}^{-2} g_{0}$ for $\delta \ll 1$, because by (5.13) the Hamiltonian vector fields of $g$ and $\tilde{g}$ are $O\left(\delta^{\mu}\right)$ close, and $\mu^{\prime}<\mu$. In particular, the rays related to $\tilde{g}$ will not hit $\partial M$ for $\delta \ll 1$. Then

$$
|Z(z, t)-\tilde{Z}(z, t)| \leq C \delta^{\mu}, \quad z \in U_{\text {out }},
$$




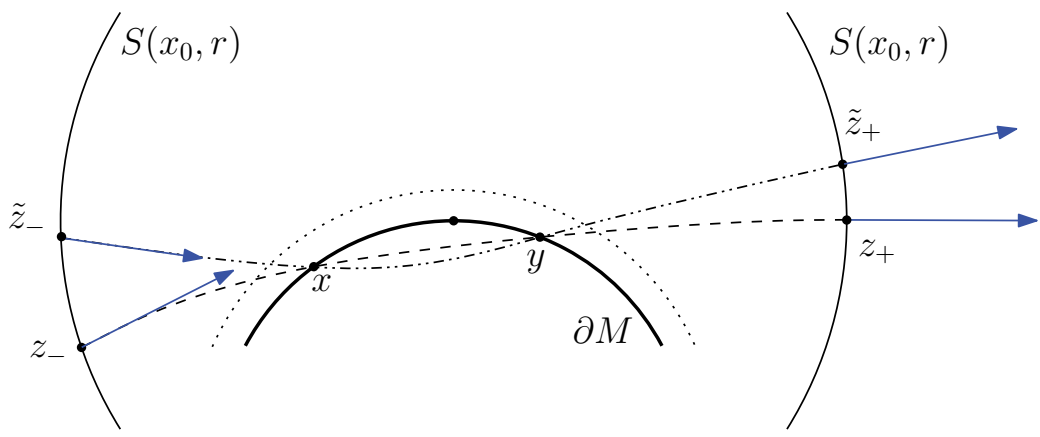

Figure 3. Illustration to the proof of Theorem 1.5. Here, $z_{ \pm}$and $\tilde{z}_{ \pm}$are actually points and codirections.

for $t$ over a compact interval. Recall that $Z(z, t)$ is the bicharacteristic issued from $z$.

The rays issued from $U_{\text {in }}$ will hit $\partial M$ at an angle at least $\delta^{\mu^{\prime} / 2} / C$, by the strict convexity. This is true, for $\delta \ll 1$, for the rays related to $\tilde{g}$ as well by the closeness of the Hamiltonians (outside $M$ ) argument. The travel times $\tilde{\ell}(z)$ of the rays in the metric $\tilde{g}$ are in general different from $\ell(z)$, but the points $\tilde{Z}(\ell(z), z)$ lie at a distance from $Z(t, \ell(z)) \in \partial_{+} S M$ which decreases with $\varepsilon$ in (1.2). Therefore, for $0<\varepsilon \ll 1, \tilde{Z}(t, z)$ with $z \in U_{\text {in }}$ would enter $M$ and leave it for $t=\ell(z)$, and it will also leave the $O\left(\delta^{\mu}\right)$ neighborhood of $M$ where we modified $\tilde{c}$. This is needed for the argument below.

By (5.15) and (5.16),

$$
\left|z_{-}-\tilde{z}_{-}\right| \leq C \delta^{\mu} / d^{2}, \quad\left|z_{+}-\tilde{z}_{+}\right| \leq C \delta^{\mu} / d^{2}, z \in U_{\text {in }} .
$$

Here, $d=d(x, y)$ with $(x, y)$ uniquely determined as the contacts of the ray trough $z$ with $\partial M$. Also, by the closeness of the Hamiltonians outside $M$, and by (5.16), the lengths of the segments of $Z(z, t)$ and $\tilde{Z}(\tilde{z}, t)$ from $z_{-}$and $\tilde{z}_{-}$, respectively, to their common point $x$, differ by $O\left(\delta^{\mu} / d^{2}\right)$; see Figure 3. The segments in $M$ differ by $O\left(\delta^{\mu} / d\right)$; see (5.14). The remaining segments, after they exit $M$ and hit $S\left(x_{0}, r\right)$, differ by $O\left(\delta^{\mu} / d^{2}\right)$ by the above argument. Therefore,

$$
|\ell(z)-\tilde{\ell}(\tilde{z})| \leq C \delta^{\mu} / d^{2}, \quad z \in U_{\text {in }}
$$

By (2.4),

$$
\begin{aligned}
\tilde{Z}\left(\ell\left(z_{-}\right), z_{-}\right) & -Z\left(\ell\left(z_{-}\right), z_{-}\right) \\
& =\int_{0}^{\ell\left(z_{-}\right)} \frac{\partial \tilde{Z}}{\partial z}\left(t-s, Z\left(s, z_{-}\right)\right)(\tilde{V}-V)\left(Z\left(s, z_{-}\right)\right) \mathrm{d} s .
\end{aligned}
$$

We replace next $\tilde{Z}\left(\ell\left(z_{-}\right), z_{-}\right)$by $\tilde{Z}\left(\tilde{\ell}\left(\tilde{z}_{-}\right), \tilde{z}_{-}\right)=\tilde{z}_{+}$modulo errors controlled by (5.18) and (5.19), so that we can get $z_{+}-z_{-}$on the left and use the fact that the latter satisfies (5.18). This would allow us to conclude that the integral is "small" in $U_{\text {in }}$. Indeed, since $Z(z, t)$ is a smooth function with uniformly bounded derivatives for $z$ and $t$ bounded, we get by (5.18) and (5.19)

$$
\left|\tilde{Z}\left(\ell\left(z_{-}\right), z_{-}\right)-\tilde{Z}\left(\tilde{\ell}\left(\tilde{z}_{-}\right), \tilde{z}_{-}\right)\right| \leq C \delta^{\mu} / d^{2}, \quad z \in U_{\text {in }} .
$$


This and (5.20) yield

$$
\begin{aligned}
& \int_{0}^{\ell\left(z_{-}\right)} \frac{\partial \tilde{Z}}{\partial z}\left(t-s, Z\left(s, z_{-}\right)\right)(\tilde{V}-V)\left(Z\left(s, z_{-}\right)\right) d s \\
&=\tilde{z}_{+}-z_{+}+O\left(\delta^{\mu} / d^{2}\right)=O\left(\delta^{\mu} / d^{2}\right), \quad z \in U_{\mathrm{in}},
\end{aligned}
$$

where, in the last step, we used the second inequality in (5.18). The factor $d(x, y)$ can be replaced by the square root of the distance between $U_{\text {in }}$ and the tangent manifold $\Sigma_{-}$, and that root is $\delta^{\mu^{\prime} / 2}$ by construction. We choose $\mu^{\prime}$ so that $\mu-$ $2 \mu^{\prime} / 2>\mu / 2$ to obtain $O\left(\delta^{\mu / 2}\right)$ in (5.22).

In $U_{\text {out }}$, the even better estimate $O\left(\delta^{\mu}\right)$ holds, without the $d^{-2}$ term, by (5.17). In $U_{0}$, we used the argument explained before to extend the estimate from $U_{\text {out }}$ to get an $O\left(\delta^{\mu}\right)+O\left(\delta^{\mu^{\prime}}\right)=O\left(\delta^{\mu^{\prime}}\right)$ estimate there. By interpolation, we get

$$
\int_{0}^{\ell\left(z_{-}\right)} \frac{\partial \tilde{Z}}{\partial z}\left(t-s, Z\left(s, z_{-}\right)\right)(\tilde{V}-V)\left(Z\left(s, z_{-}\right)\right) \mathrm{d} s=O\left(\delta^{\mu / 2}\right) \quad \text { in } C^{1}(U) .
$$

Now, by (2.4), (2.11), and (2.12) we can write the left-hand side of (5.23) in the form $\tilde{J}(f, \partial f)$ and complete the proof of Theorem 5.1 by applying Proposition 3.3 .

5.3. Global stability. For the purpose of the next theorem, we will extend and slightly generalize the foliation condition to compact submanifolds $M_{0}$ of $M$. Let, as before, $\tilde{M}$ be a neighborhood of $M$, and extend $c$ smoothly there. Note that the tilde over $M$ is not an indication that it is related to $\tilde{c}$.

Definition 5.1. Let $M_{0} \subset M$ be compact. We say that $M_{0}$ can be foliated by strictly convex hypersurfaces if there exists a smooth function $\rho: \tilde{M} \rightarrow[0, \infty)$ which level sets $\Sigma_{t}=\rho^{-1}(t), t \leq T$ with some $T>0$, restricted to $M_{0}$, are strictly convex viewed from $\rho^{-1}((0, t))$ for $g$; $d \rho$ is non-zero on these level sets, $\Sigma_{0} \cap M=\emptyset$ and $M_{0} \subset \rho^{-1}([0, T])$.

Note that this definition is not equivalent to Definition 1.1 when $M_{0}=M$ because in Definition 1.1, we allow $M \backslash \rho^{-1}([0, T])$ to be non-empty (but with an empty interior). Indeed, for uniqueness, proving $c=\tilde{c}$ outside such a set suffices since $c$ and $\tilde{c}$ are at least continuous. For stability, however, it is convenient to assume that this set is empty.

We show next that the foliation condition implies non-trapping.

Proposition 5.1. If $M_{0}$ can be foliated by strictly convex hypersurfaces, then any maximal geodesic in $M_{0}$ is of finite length.

Proof. Assume that $s \mapsto \gamma(s)$ exists for $s \in(0, \infty)$ and stays in $M_{0}$; and in particular, it never reaches $\partial M_{0}$ for $s>0$. The function $f(s):=\rho \circ \gamma(s)$ cannot have more than one critical point as a consequence of the implication $f^{\prime}(s)=0 \Longrightarrow f^{\prime \prime}(s)<0$. By shifting the $s$ variable, we can always assume that the possible critical point is negative. Then $f$ is a strictly decreasing function for $s>0$. On the other hand, it is bounded by below, so it has a limit $\hat{\tau} \geq 0$, as $s \rightarrow \infty$, which is also its infimum. By compactness, there exists a sequence $s_{j} \rightarrow \infty$ (we can start with $s_{j}=j$ and take a subsequence) so that $\left(x_{j}, v_{j}\right):=(\gamma(s), \dot{\gamma}(s))$ converges to some $(\hat{x}, \hat{v}) \in S M_{0}$. Next, $\rho\left(x_{j}\right) \searrow \hat{\tau}$. The limit $\hat{v}$ must be tangent to $\Sigma_{\hat{\tau}}$ at $\hat{x}$, because we can easily obtain a contradiction with the strict convexity if it is not. Now, by the strict convexity of $\Sigma_{\hat{\tau}}$ again, there exists $\delta>0$ so that $\gamma_{\hat{x}, \hat{v}}(s)$ would hit $\Sigma_{\hat{\tau}-\delta}$ for some positive 
time. This property is preserved under a small perturbation of $(\hat{x}, \hat{v})$ and therefore applies to $\left(x_{j}, v_{j}\right)$ for $j \gg 1$. This, however, contradicts the choice of $\hat{\tau}$.

In particular, we get the following.

Corollary 5.1. Assume that $M=M_{0} \cup M_{1}$, where $M_{0}$ can be foliated by strictly convex hypersurfaces, and $M_{1}$ is non-trapping. Then $M$ is non-trapping as well.

Note that $M_{1}$ can be a point, or a small neighborhood of a point, which happens if the level surfaces of $\rho=c>0$ are diffeomorphic to spheres but $\rho=0$ degenerates into a point. Another example is when $M_{1}$ is simple; see also the remarks following Theorem 1.4.

The $C^{k}$ norm below is defined in a fixed finite atlas of local coordinate charts. In the same way we define $\operatorname{dist}(L, \tilde{L})$ and its $C(D)$ norm: in any coordinate system we can just take the supremum of $L-\tilde{L}$ and then the maximum over all charts. They can be defined in an invariant way, in principle but we do not do that for the sake of simplicity.

Theorem 5.2. Assume that $M_{0} \subset M$ can be foliated by strictly convex hypersurfaces for $g=c^{-2} g_{0}$. Let $D \subset \partial_{-} S M$ be a neighborhood of the compact set of all $\beta \in \partial_{-} S M \cap \partial_{-} S M_{0}$ consisting of the initial points of all geodesics $\gamma_{\beta}$ tangent to the intersections of the strictly convex hypersurfaces with $M_{0}$. Then with $k, \mu, c_{0}$, $c, \tilde{c}, \varepsilon_{0}$, and $A$ as in Theorem 1.5, we have the stability estimate

$$
\|c-\tilde{c}\|_{C^{2}\left(M_{0}\right)} \leq C\|\operatorname{dist}(L, \tilde{L})\|_{C(D)}^{\mu}
$$

for $c$, $\tilde{c}$ satisfying (1.2).

Proof. We will show first that the estimate in Theorem 1.5, near a boundary point $p$, can be written in the form (5.24). For $x$ and $y$ on $\partial M$, both close to the fixed point $p$, let $\gamma_{0}:[0,1] \mapsto \partial M$ be the boundary geodesic connecting $x$ (for $t=0$ ) and $y$ (for $t=1$ ). Set $v(s)=\exp _{x}^{-1} \gamma_{0}(s)$, where exp is the interior exponential map. Then $(y, w)=L\left(x, v(1) /|v(1)|_{g}\right)$; set $(\tilde{y}, \tilde{w})=\tilde{L}\left(x, v(1) /|v(1)|_{\tilde{g}}\right)$. Set

$$
\delta=\|\operatorname{dist}(L, \tilde{L})\|_{C(D)} .
$$

Then $|y-\tilde{y}| \leq \delta$. On the other hand, in the notation following (1.1), given by $L(x, v)=(y, w)$,

$$
\frac{\mathrm{d}}{\mathrm{d} s} d\left(x, \gamma_{0}(s)\right)=\left\langle w^{\prime}(s), \dot{\gamma}_{0}(s)\right\rangle_{g},
$$

where $w^{\prime}(s)$ is the tangential component of $w(s)$, the second component of $L\left(x, v(s) /|v(s)|_{g}\right)$. We also have

$$
\frac{\mathrm{d}}{\mathrm{d} s} \tilde{d}\left(x, \gamma_{0}(s)\right)=\left\langle\tilde{w}^{\prime}(s), \dot{\gamma}_{0}(s)\right\rangle_{\tilde{g}},
$$

where $\tilde{w}(s)$ is the second component of $\tilde{L}\left(x, v(s) /|v(s)|_{\tilde{g}}\right)$. Integrate the difference of (5.26) and (5.27) w.r.t. $s$ from 0 to 1 , and use (5.25) and the fact that $c=$ $\tilde{c}$ on $\partial M$ near $x$ to get $d(x, y)-\tilde{d}(x, \tilde{y})=O(\delta)$ (actually, we get $O(\delta) d(x, y)$ ). Note that this also proves that $\ell-\tilde{\ell}=O(\delta)$ near $S_{x_{0}} M$. We remark that in the inductive step below, we will only have that $c$ and $\tilde{c}$ are $O\left(\delta^{\mu}\right)$ close on $\partial M$ near $x$, instead of being equal. Then we would get $O\left(\delta^{\mu}\right)$ instead of $O(\delta)$. So we get now 
$d^{2}(x, y)-\tilde{d}^{2}(x, \tilde{y})=O(\delta)$ as well. Since $d^{2}$ and $\tilde{d}^{2}$ are smooth and $|y-\tilde{y}| \leq \delta$, we get

$$
d^{2}(x, y)-\tilde{d}^{2}(x, y)=O(\delta)
$$

The constant in this estimate depends on the a priori bound $A$ in (1.2). We can therefore apply Theorem 5.1 to estimate the jet of $c-\tilde{c}$ at $\partial M$ of any finite order. We then extend $c$ and $\tilde{c}$ in a neighborhood of $\partial M_{0} \cap \partial M$ so that $c$ and $\tilde{c}$ are $O\left(\delta^{\mu}\right)$ there, as in (5.13).

For any geodesic in $M_{0}$ issued from $D$, we extend it to $\tilde{M}$ and push the scattering relations $L$ and $\tilde{L}$ to $\partial \tilde{M}$. We show below that we have $\operatorname{dist}(L, \tilde{L})=O\left(\delta^{\mu}\right)$ for the so extended lens relations, and a similar estimate for $\ell-\tilde{\ell}$. We choose $U_{0}, U_{\text {in }}$, and $U_{\text {out }}$ as the proof of Theorem 5.1 but this time $U_{0}$ is an $O\left(\delta^{\mu}\right)$ neighborhood of $\Sigma$ (rather than $O\left(\delta^{\mu^{\prime}}\right)$, because the singular factor $1 / d^{2}$ is not present anymore). For any $(x, v) \in U_{\text {in }}$, let $z_{-}, \tilde{z}_{-}$be the points (in the phase space) of contact with the geodesic issued from $(x, v)$ for negative "times" with $\partial \tilde{M}$ w.r.t. the metrics $g$ and $\tilde{g}$, respectively. We define $z_{+}, \tilde{z}_{+}$in a similar way (see Figure 4), where, in this case, $\Sigma_{i}=\partial M$ and $\Omega_{i}=M_{0}$. Then $z_{-}-\tilde{z}_{-}=O\left(\delta^{\mu}\right)$ (the difference makes sense in local coordinates only) because we have the same for $c-\tilde{c}$ in $C^{2}$. We have an $O(\delta)$ bound of $y-\tilde{y}$, and of the difference of the corresponding directions there, by the closeness of the scattering relations. Next, $z_{+}-\tilde{z}_{+}=O\left(\delta^{\mu}\right)$ as well. Therefore, $\operatorname{dist}\left(L\left(z_{-}\right), \tilde{L}\left(\tilde{z}_{-}\right)\right)=O\left(\delta^{\mu}\right)$. Since $L$ and $\tilde{L}$ are smooth with uniformly bounded first derivatives, we also get $\operatorname{dist}\left(L\left(z_{-}\right), \tilde{L}\left(z_{-}\right)\right)=O\left(\delta^{\mu}\right)$, for all $z_{-} \in U_{\text {in }}$. We also have $\ell\left(z_{-}\right)-\tilde{\ell}\left(z_{-}\right)=O\left(\delta^{\mu}\right)$ as shown in the paragraph following (5.27). In this step, we are no longer working near a fixed point on $\partial M$, and for almost tangential directions, the requirement for $U_{\text {in }}$ is that the geodesic issued from it are in the set required by the theorem but that set is a distance at least $\delta^{\mu}$ from the tangential set $\Sigma$. In $U_{\text {out }}$ and $U_{0}$, we argue as above.

Therefore, we have now that $\|\operatorname{dist}(L, \tilde{L})\|_{C\left(D_{0}\right)}=O\left(\delta^{\mu}\right)$, where $L, \tilde{L}$, and $D_{0}$ are defined as $L, \tilde{L}$, and $D$ but with $\partial M$ replaced by $\partial \tilde{M}$. We have the same for $\ell-\tilde{\ell}$ as well. The advantage of this is that the geodesics issued from $D_{0}$ hitting $\operatorname{supp}(c-\tilde{c})$ are never tangent to $\partial \tilde{M}$, and we actually have a uniform lower bound on the angle they make with $\partial \tilde{M}$. Thus we reduced the analysis to the case when $c-\tilde{c}$ is a priori known to be supported in the interior of $M$.

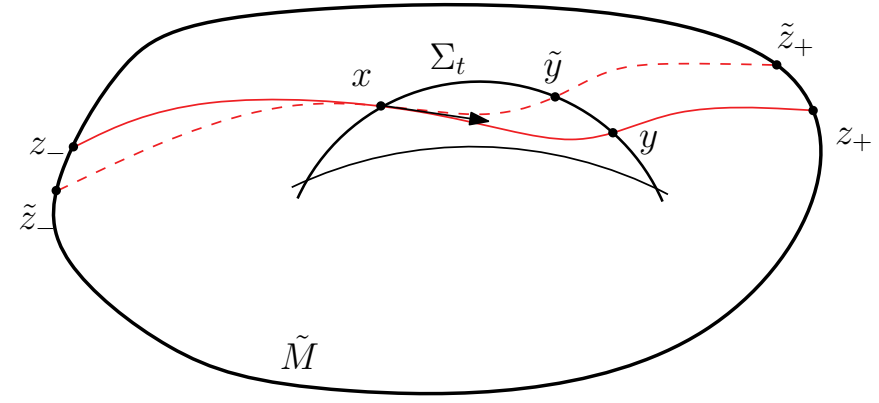

Figure 4. Illustration to the proof of Theorem 5.2

We then apply a layer stripping argument finite number of times; see also the introduction in [49]. For each $\Sigma_{t}$, assuming the estimate outside $\Sigma_{t}$, we can choose 
an appropriate lens shaped domain near each point on $\Sigma_{t}$ and the artificial boundary close enough to it; see Figure 4 . The $O\left(\delta^{\mu}\right)$ closeness of $L$ and $\tilde{L}$, and $\ell$ and $\tilde{\ell}$, implies the same for $c$ and $\tilde{c}$ by the arguments using (5.23). We do this for a finite number of points on $\Sigma_{t}$ by a compactness argument to prove the estimate in $\rho^{-1}(0, t+\epsilon)$ for some $\epsilon>0$. We then cover $M_{0}$ with a finite set $\Sigma_{i}:=\rho^{-1}\left(t_{i}\right), M_{i}:=\rho^{-1}\left(-\infty, t_{i}\right)$, and finish the proof with finitely many steps.

\section{Herglotz And Wieckert And Zoeppritz speeds ARE Lens Rigid}

We revisit the Herglotz [14] and Wieckert and Zoeppritz [51] class of speeds. Let $M=B(0, R), R>0$, be the ball in $\mathbb{R}^{n}, n \geq 3$, centered at the origin with radius $R>0$. The background metric $g_{0}$ in this section is the Euclidean one. Let $0<c(x)$ be smooth in $B(0, R)$. Assume that $c$ satisfies the Herglotz and Wieckert and Zoeppritz condition

$$
\frac{\partial}{\partial r} \frac{r}{c(r)}>0, \quad \text { for } 0<r=|x| \leq R,
$$

where $\frac{\partial}{\partial r}=\frac{x}{|x|} \cdot \partial_{x}$ is the radial derivative. We do not assume that $c$ is radial, i.e., that it depends on $r=|x|$ only. We show below that (6.1) is, in fact a foliation condition.

Proposition 6.1. The Herglotz and Wieckert and Zoeppritz condition (6.1) is equivalent to the condition that the Euclidean spheres $S_{r}=\{|x|=r\}$ are strictly convex in the metric $c^{-2} \mathrm{~d} x^{2}$ for $0<r \leq R$.

Proof. This proposition is essentially proved in [47, Proposition 7.1]. We will show first that the strict convexity condition of an oriented hypersurface $S$ (positive second fundamental form) is equivalent to the following: (A) if $G$ is the generator of the geodesic flow, and if $\rho$ is a defining function of $S$ positive on the "positive" side of $S$, then $G^{2} \rho>0$ when $G \rho=\rho=0$ on a non-zero energy level. In semigeodesic coordinates $\left(x^{\prime}, x^{n}\right)$ with $x^{n}>0$ on the "positive" side of $S$, we have $\rho=x^{n} \rho^{\prime}$ with $\rho^{\prime}>0$ on $S$. Since

$$
G=\xi^{j} \partial_{x^{j}}-\Gamma_{i j}^{k} \xi^{i} \xi^{j} \partial_{\xi_{k}},
$$

we have $G \rho=\xi^{n}+x^{n} G \rho^{\prime}$. On $S, G \rho=\xi^{n}$; therefore, (A) can be reformulated as follows: $x^{n}=\xi^{n}=0$ and $\xi \neq 0$ imply $G^{2} \rho>0$. Differentiating $G \rho$ again, we see that under those conditions, $G^{2} \rho=-\Gamma_{\alpha \beta}^{n} \xi^{\alpha} \xi^{\beta}$, where the Greek indices run from 1 to $n-1$. This is well known and follows directly from the definition to be the second fundamental form corresponding to the orientation $x^{n}>0$. Then strict convexity, viewed from $\rho>0$ is equivalent to a negative second fundamental form. For manifolds with a boundary we study $\rho>0$ in the interior of $M$, and therefore convexity viewed from the exterior means a positive second fundamental form.

We can take $\rho=r^{2} / 2-r_{0}^{2} / 2=|x|^{2} / 2-r_{0}^{2} / 2$ as a defining function of the sphere $S_{r_{0}}$ for $x \neq 0$. Then $G \rho=\xi \cdot x$. Next, $G^{2} \rho=|\xi|^{2}-\Gamma_{i j}^{k} \xi^{i} \xi^{j} x^{k}$ and on the energy level $c^{-2}|\xi|^{2}=1$, we have $G^{2} \rho=c^{2}-\Gamma_{i j}^{k} \xi^{i} \xi^{j} x^{k}$. Here and below, we still have summation w.r.t. $k$ even though both indices are upper. A direct computation shows that

$$
\Gamma_{i j}^{k}=\frac{1}{2} c^{2}\left(\delta_{j}^{k} \partial_{x^{i}}+\delta_{i}^{k} \partial_{x^{j}}-\delta_{i j} \partial_{x^{k}}\right) c^{-2}
$$


with $c=c(|x|)$, and therefore,

$$
\begin{aligned}
\Gamma_{i j}^{k} \xi^{i} \xi^{j} x^{k} & =-c^{-1}\left(2 \xi^{k} x^{k} \xi^{i} \partial_{x^{i}}-|\xi|^{2} x^{k} \partial_{x^{k}}\right) c \\
& =-c^{-1}\left(2(\xi \cdot x) \xi \cdot \partial_{x}-|\xi|^{2} x \cdot \partial_{x}\right) c .
\end{aligned}
$$

Therefore, on the unit energy level and for $G \rho=0$, strict convexity is equivalent to

$$
c-x \cdot \partial_{x} c>0
$$

i.e., $r \partial_{r} c<c$, which is equivalent to (6.1). Note that the computations in 47 , Proposition 7.1] are done in the cotangent bundle and are somewhat shorter.

Corollary 6.1. Speeds satisfying (6.1) are lens rigid in $B(0, R)$ in the sense of Theorem 1.4 .

Note that we only require $c$ in Theorem 1.4 to satisfy (6.1). Then any other speed $\tilde{c}$ for which $\partial B(0, R)$ is still strictly convex and $\tilde{c}=c$ on $\partial B(0, R)$ with the same scattering relation is equal to $c$. This extends the Herglotz 14 and the Wieckert and Zoeppritz [51] results to not necessarily radial speeds $c$ satisfying (6.1).

If (6.1) holds in the shell $R_{0}<|x|<R$ only, with some $0<R_{0}<R$, then we get lens rigidity in the shell and we only need to use the scattering relation for geodesics staying in it. The speed $c$ does not even need to be defined in $|x|<R_{0}$. We will skip the details.

\section{ACKNOWLEDGMENTS}

The authors would like to thank Christopher Croke for pointing out an error in the formulation of Theorem 1.4 in an earlier version of the paper and for helpful comments.

\section{REFERENCES}

[1] Gang Bao and Hai Zhang, Sensitivity analysis of an inverse problem for the wave equation with caustics, J. Amer. Math. Soc. 27 (2014), no. 4, 953-981, DOI 10.1090/S0894-0347-201400787-6. MR 3230816

[2] G. Besson, G. Courtois, and S. Gallot, Entropies et rigidités des espaces localement symétriques de courbure strictement négative (French), Geom. Funct. Anal. 5 (1995), no. 5, 731-799, DOI 10.1007/BF01897050. MR1354289 (96i:58136)

[3] Dmitri Burago and Sergei Ivanov, Boundary rigidity and filling volume minimality of metrics close to a flat one, Ann. of Math. (2) 171 (2010), no. 2, 1183-1211, DOI 10.4007/annals.2010.171.1183. MR2630062 (2011d:53079)

[4] Christopher Croke, Scattering rigidity with trapped geodesics, Ergodic Theory Dynam. Systems 34 (2014), no. 3, 826-836, DOI 10.1017/etds.2012.164. MR3199795

[5] K. C. Creager, Anisotropy of the inner core from differential travel times of the phases PKP and PKIPK, Nature 356 (1992), 309-314.

[6] Christopher B. Croke, Rigidity for surfaces of nonpositive curvature, Comment. Math. Helv. 65 (1990), no. 1, 150-169, DOI 10.1007/BF02566599. MR.1036134 (91d:53056)

[7] Christopher B. Croke, Rigidity and the distance between boundary points, J. Differential Geom. 33 (1991), no. 2, 445-464. MR.1094465 (92a:53053)

[8] Christopher B. Croke, Rigidity theorems in Riemannian geometry, Geometric methods in inverse problems and PDE control, IMA Vol. Math. Appl., vol. 137, Springer, New York, 2004, pp. 47-72, DOI 10.1007/978-1-4684-9375-7_4. MR2169902(2007c:53052) 
[9] Christopher B. Croke and Nurlan S. Dairbekov, Lengths and volumes in Riemannian manifolds, Duke Math. J. 125 (2004), no. 1, 1-14, DOI 10.1215/S0012-7094-04-12511-4. MR2097355 (2005k:53045)

[10] Christopher B. Croke and Bruce Kleiner, Conjugacy and rigidity for manifolds with a parallel vector field, J. Differential Geom. 39 (1994), no. 3, 659-680. MR.1274134 (95a:53064)

[11] Nurlan S. Dairbekov, Integral geometry problem for nontrapping manifolds, Inverse Problems 22 (2006), no. 2, 431-445, DOI 10.1088/0266-5611/22/2/003. MR2216407 (2007i:53084)

[12] C. Guillarmou, Lens rigidity for manifolds with hyperbolic trapped set, available at arXiv: 1412.1760

[13] Mikhael Gromov, Filling Riemannian manifolds, J. Differential Geom. 18 (1983), no. 1, 1-147. MR697984(85h:53029)

[14] G. Herglotz, Uber die Elastizitaet der Erde bei Beruecksichtigung ihrer variablen Dichte, Z. Math. Phys. 52 (1905), 275-299.

[15] L. Hörmander, The analysis of linear partial differential operators, Vol. 1-4, Springer-Verlag, Berlin, 1983.

[16] Sergei Ivanov, Volume comparison via boundary distances, Proceedings of the International Congress of Mathematicians. Volume II, Hindustan Book Agency, New Delhi, 2010, pp. 769-784. MR2827818 (2012g:53064)

[17] Venkateswaran P. Krishnan, A support theorem for the geodesic ray transform on functions, J. Fourier Anal. Appl. 15 (2009), no. 4, 515-520, DOI 10.1007/s00041-009-9061-5. MR2549942 (2010i:53146)

[18] Venkateswaran P. Krishnan and Plamen Stefanov, A support theorem for the geodesic ray transform of symmetric tensor fields, Inverse Probl. Imaging 3 (2009), no. 3, 453-464, DOI 10.3934/ipi.2009.3.453. MR2557914 (2010m:53110)

[19] Matti Lassas, Vladimir Sharafutdinov, and Gunther Uhlmann, Semiglobal boundary rigidity for Riemannian metrics, Math. Ann. 325 (2003), no. 4, 767-793, DOI 10.1007/s00208-0020407-4. MR.1974568 (2004c:53053)

[20] Richard B. Melrose, Spectral and scattering theory for the Laplacian on asymptotically Euclidian spaces, Spectral and scattering theory (Sanda, 1992), Lecture Notes in Pure and Appl. Math., vol. 161, Dekker, New York, 1994, pp. 85-130. MR.1291640 (95k:58168)

[21] René Michel, Sur la rigidité imposée par la longueur des géodésiques (French), Invent. Math. 65 (1981/82), no. 1, 71-83, DOI 10.1007/BF01389295. MR636880 (83d:58021)

[22] R. G. Muhometov, The reconstruction problem of a two-dimensional Riemannian metric, and integral geometry (Russian), Dokl. Akad. Nauk 232 (1977), no. 1, 32-35. MR0431074 (55 \#4076)

[23] R. G. Muhometov, On a problem of reconstructing Riemannian metrics (Russian), Sibirsk. Mat. Zh. 22 (1981), no. 3, 119-135, 237. MR621466 (82m:53071)

[24] R. G. Muhometov and V. G. Romanov, On the problem of finding an isotropic Riemannian metric in an n-dimensional space (Russian), Dokl. Akad. Nauk 243 (1978), no. 1, 41-44. MR.511273 (81a:53059)

[25] Cesare Parenti, Operatori pseudo-differenziali in $R^{n}$ e applicazioni, Ann. Mat. Pura Appl. (4) 93 (1972), 359-389. MR0437917 (55 \#10838)

[26] Gabriel P. Paternain, Mikko Salo, and Gunther Uhlmann, Tensor tomography on surfaces, Invent. Math. 193 (2013), no. 1, 229-247, DOI 10.1007/s00222-012-0432-1. MR3069117

[27] Gabriel P. Paternain, Mikko Salo, and Gunther Uhlmann, The attenuated ray transform for connections and Higgs fields, Geom. Funct. Anal. 22 (2012), no. 5, 1460-1489, DOI 10.1007/s00039-012-0183-6. MR2989440

[28] Gabriel P. Paternain, Mikko Salo, and Gunther Uhlmann, Tensor tomography: progress and challenges, Chin. Ann. Math. Ser. B 35 (2014), no. 3, 399-428, DOI 10.1007/s11401-0140834-z. MR 3200025

[29] Leonid Pestov and Gunther Uhlmann, Two dimensional compact simple Riemannian manifolds are boundary distance rigid, Ann. of Math. (2) 161 (2005), no. 2, 1093-1110, DOI 10.4007/annals.2005.161.1093. MR2153407(2006c:53038)

[30] L. N. Pestov and V. A. Sharafutdinov, Integral geometry of tensor fields on a manifold of negative curvature (Russian), Sibirsk. Mat. Zh. 29 (1988), no. 3, 114-130, 221, DOI 10.1007/BF00969652; English transl., Sib. Math. J. 29 (1988), no. 3, 427-441 (1989). MR.953028(89k:53066) 
[31] Akhil Ranjan and Hemangi Shah, Convexity of spheres in a manifold without conjugate points, Proc. Indian Acad. Sci. Math. Sci. 112 (2002), no. 4, 595-599, DOI 10.1007/BF02829692. MR:1941895 (2003h:53047)

[32] Vladimir Sharafutdinov, Michal Skokan, and Gunther Uhlmann, Regularity of ghosts in tensor tomography, J. Geom. Anal. 15 (2005), no. 3, 499-542, DOI 10.1007/BF02930983. MR2190243 (2006m:58034)

[33] V. A. Sharafutdinov, Integral geometry of tensor fields, Inverse and Ill-posed Problems Series, VSP, Utrecht, 1994. MR1374572(97h:53077)

[34] V. A. Sharafutdinov, Integral geometry of a tensor field on a surface of revolution (Russian, with Russian summary), Sibirsk. Mat. Zh. 38 (1997), no. 3, 697-714, iv, DOI 10.1007/BF02683847; English transl., Sib. Math. J. 38 (1997), no. 3, 603-620. MR.1457488 (98f:53067)

[35] V. A. Sharafutdinov, A problem in integral geometry in a nonconvex domain (Russian, with Russian summary), Sibirsk. Mat. Zh. 43 (2002), no. 6, 1430-1442, DOI 10.1023/A:1021189922555; English transl., Sib. Math. J. 43 (2002), no. 6, 1159-1168. MR.1946241 (2003i:53112)

[36] Vladimir Sharafutdinov, Variations of Dirichlet-to-Neumann map and deformation boundary rigidity of simple 2-manifolds, J. Geom. Anal. 17 (2007), no. 1, 147-187, DOI 10.1007/BF02922087. MR2302878(2008f:58024)

[37] M. A. Šubin, Pseudodifferential operators in $R^{n}$ (Russian), Dokl. Akad. Nauk 196 (1971), 316-319. MR0273463 (42 \#8341)

[38] Plamen Stefanov, Microlocal approach to tensor tomography and boundary and lens rigidity, Serdica Math. J. 34 (2008), no. 1, 67-112. MR2414415 (2009d:53051)

[39] Plamen Stefanov and Gunther Uhlmann, Rigidity for metrics with the same lengths of geodesics, Math. Res. Lett. 5 (1998), no. 1-2, 83-96, DOI 10.4310/MRL.1998.v5.n1.a7. MR 1618347 (99e:53059)

[40] Plamen Stefanov and Gunther Uhlmann, Stability estimates for the X-ray transform of tensor fields and boundary rigidity, Duke Math. J. 123 (2004), no. 3, 445-467, DOI 10.1215/S00127094-04-12332-2. MR2068966 (2005h:53130)

[41] Plamen Stefanov and Gunther Uhlmann, Boundary rigidity and stability for generic simple metrics, J. Amer. Math. Soc. 18 (2005), no. 4, 975-1003, DOI 10.1090/S0894-0347-05-004947. MR2163868 (2006h:53031)

[42] Plamen Stefanov and Gunther Uhlmann, Boundary and lens rigidity, tensor tomography and analytic microlocal analysis, Algebraic analysis of differential equations from microlocal analysis to exponential asymptotics, Springer, Tokyo, 2008, pp. 275-293, DOI 10.1007/9784-431-73240-2_23. MR2758914 (2012c:58046)

[43] Plamen Stefanov and Gunther Uhlmann, Integral geometry on tensor fields on a class of non-simple Riemannian manifolds, Amer. J. Math. 130 (2008), no. 1, 239-268, DOI 10.1353/ajm.2008.0003. MR2382148(2009e:53051)

[44] Plamen Stefanov and Gunther Uhlmann, Local lens rigidity with incomplete data for a class of non-simple Riemannian manifolds, J. Differential Geom. 82 (2009), no. 2, 383-409. MR.2520797(2011d:53081)

[45] Plamen Stefanov and Gunther Uhlmann, Recovery of a source term or a speed with one measurement and applications, Trans. Amer. Math. Soc. 365 (2013), no. 11, 5737-5758, DOI 10.1090/S0002-9947-2013-05703-0. MR3091263

[46] Plamen Stefanov and Gunther Uhlmann, The geodesic X-ray transform with fold caustics, Anal. PDE 5 (2012), no. 2, 219-260, DOI 10.2140/apde.2012.5.219. MR2970707

[47] P. Stefanov and G. Uhlmann, Multi-wave methods via ultrasound, in Inside Out, Vol. 60, MSRI Publications, Cambridge, UK, 2009.

[48] Hans Triebel, Interpolation theory, function spaces, differential operators, North-Holland Mathematical Library, vol. 18, North-Holland Publishing Co., Amsterdam-New York, 1978. MR.503903(80i:46032b)

[49] G. Uhlmann and A. Vasy, The inverse problem for the local geodesic ray transform, available at arXiv:1210.2084. To appear in Invent. Math.

[50] James Vargo, A proof of lens rigidity in the category of analytic metrics, Math. Res. Lett. 16 (2009), no. 6, 1057-1069, DOI 10.4310/MRL.2009.v16.n6.a13. MR2576693(2011a:53068) 
[51] E. Wiechert and K. Zoeppritz, Über Erdbebenwellen, Nachr. Koenigl. Geselschaft Wiss. Göttingen 4 (1907), 415-549.

[52] H. Zhou, The inverse problem for the local ray transform, available at arXiv:1304.7023.

Department of Mathematics, Purdue University, West Lafayette, Indiana 47907

E-mail address: Plamen-Stefanov@purdue.edu

Department of Mathematics, University of Washington, Seattle, Washington 98195 and Department of Mathematics, University of Helsinki, Finland Fi-00014

E-mail address: gunther@math.washington.edu

Department of Mathematics, Stanford University, Stanford, California 94305

E-mail address: andras@stanford.edu 